\title{
Singular fibers of stable maps and signatures of 4-manifolds
}

\author{
OSAMU SAEKI \\ TAKAHIRO YAMAMOTO
}

\begin{abstract}
We show that for a $C^{\infty}$ stable map of an oriented 4-manifold into a 3-manifold, the algebraic number of singular fibers of a specific type coincides with the signature of the source 4-manifold.

57R45; 57N13, 58K30, 58K15
\end{abstract}

\section{Introduction}

In [24] the first named author developed the theory of singular fibers of generic differentiable maps between manifolds of negative codimension. Here, the codimension of a map $f: M \rightarrow N$ between manifolds is defined to be $k=\operatorname{dim} N-\operatorname{dim} M$. For $k \geq 0$, the fiber over a point in $N$ is a discrete set of points, as long as the map is generic enough, and we can study the topology of such maps by using the well-developed theory of multi-jet spaces (see, for example, the article [13] by Mather). However, in the case where $k<0$, the fiber over a point is no longer a discrete set, and is a complex of positive dimension $-k$ in general. This means that the theory of multi-jet spaces is not sufficient any more, and in [24] we have seen that the topology of singular fibers plays an essential role in such a study.

In [24], as an explicit and important example of the theory of singular fibers, $C^{\infty}$ stable maps of closed orientable 4-manifolds into 3-manifolds were studied and their singular fibers were completely classified up to the natural equivalence relation, called the $C^{\infty}$ (or $C^{0}$ ) right-left equivalence (for a precise definition, see Section 2 of the present paper). Furthermore, it was proved that the number of singular fibers of a specific type (in the terminology of [24], singular fibers of type $\mathrm{III}^{8}$ ) of such a map is congruent modulo two to the Euler characteristic of the source 4-manifold (see [24, Theorem 5.1] and also Corollary 5.6 of the present paper).

In this paper, we will give an "integral lift" of this modulo two Euler characteristic formula. More precisely, we consider $C^{\infty}$ stable maps of oriented 4-manifolds into 3 -manifolds, and we give a sign +1 or -1 to each of its III $^{8}$ type fiber, using the orientation of the source 4 -manifold. Then we show that the algebraic number of III $^{8}$ 
type fibers coincides with the signature of the source oriented 4-manifold (Theorem 5.5).

For certain Lefschetz fibrations, similar signature formulas have already been proved by Matsumoto [15; 16], Endo [6], etc. Our formula can be regarded as their analogue from the viewpoint of singularity theory of generic differentiable maps. The most important difference between Lefschetz fibrations and generic differentiable maps is that not all manifolds can admit a Lefschetz fibration, while every manifold admits a generic differentiable map. (In fact, a single manifold admits a lot of generic differentiable maps.) Furthermore, it is known that similar signature formulas do not hold for arbitrary Lefschetz fibrations, since there exist oriented surface bundles over oriented surfaces with nonzero signatures (see Meyer [17]). In this sense, our formula is more general (see Remark 7.7). Our proof of the formula is based on the abundance of such generic maps in some sense.

More precisely, our proof of the formula goes as follows. We first define the notion of a chiral singular fiber (for a precise definition, see Section 2). Roughly speaking, if a fiber can be transformed to its "orientation reversal" by an orientation preserving homeomorphism of the source manifold, then we call it an achiral fiber; otherwise, a chiral fiber. On the other hand, we classify singular fibers of proper $C^{\infty}$ stable maps of orientable 5-manifolds into 4-manifolds by using methods developed in [24]. Then, for proper $C^{\infty}$ stable maps of oriented 4-manifolds into 3-manifolds, and those of oriented 5-manifolds into 4-manifolds, we determine those singular fibers in the classification lists which are chiral. Furthermore, for each chiral singular fiber that appears discretely, we define its sign $(= \pm 1)$.

Let us consider two $C^{\infty}$ stable maps of 4-manifolds into a 3-manifold which are oriented bordant. Then by using a generic bordism between them, which is a generic differentiable map of a 5 -manifold into a 4 -manifold, and by looking at the III $^{8}$-fiber locus in the target 4-manifold, we show the oriented bordism invariance of the algebraic numbers of III $^{8}$ type fibers of the original stable maps of 4-manifolds. Finally, we verify our formula for an explicit example of a stable map of an oriented 4-manifold with signature +1 . (In fact, this final step is not so easy and needs a careful analysis.) Combining all these, we will prove our formula.

The paper is organized as follows. In Section 2 we give some fundamental definitions concerning singular fibers of generic differentiable maps, among which is the notion of a chiral singular fiber. In Section 3 we recall the classification of singular fibers of proper $C^{\infty}$ stable maps of orientable 4-manifolds into 3-manifolds obtained in [24]. In Section 4 we present the classification of singular fibers of proper $C^{\infty}$ stable maps of orientable 5-manifolds into 4-manifolds. In Section 5 we determine those 
singular fibers in the classification lists which are chiral. Furthermore, for each chiral singular fiber that appears discretely, we define its sign by using the orientation of the source manifold. In Section 6 we prove the oriented bordism invariance of the algebraic number of $\mathrm{III}^{8}$ type fibers. This is proved by looking at the adjacencies of the chiral singular fiber loci in the target manifold. In Section 7 we investigate the explicit example of a $C^{\infty}$ stable map of an oriented 4-manifold into a 3-manifold constructed in [24]. In order to calculate the signature of the source 4-manifold, we will compute the self-intersection number of the surface of definite fold points by using normal sections coming from the surface of indefinite fold points. This procedure needs some technical details so that this section will be rather long. By combining the result of Section 6 with the computation of the example, we prove our main theorem. Finally in Section 8, we define the universal complex of chiral singular fibers for proper $C^{\infty}$ stable maps of 5-manifolds into 4-manifolds and compute its third cohomology group. This will give an interpretation of our formula from the viewpoint of the theory of singular fibers of generic differentiable maps as developed in [24].

Throughout the paper, all manifolds and maps are differentiable of class $C^{\infty}$. The symbol "@" denotes an appropriate isomorphism between algebraic objects. For a space $X$, the symbol "id $X$ " denotes the identity map of $X$.

The authors would like to express their thanks to András Szúcs for drawing their attention to the work of Conner-Floyd, and to Go-o Ishikawa for his invaluable comments and encouragement. They would also like to thank the referee for helpful comments. The first named author has been supported in part by Grant-in-Aid for Scientific Research (No. 16340018), Japan Society for the Promotion of Science.

\section{Preliminaries}

Let us begin with some fundamental definitions. For some of the definitions, refer to [24].

Definition 2.1 Let $M_{i}$ be smooth manifolds and $A_{i} \subset M_{i}$ be subsets, $i=0,1$. A continuous map $g: A_{0} \rightarrow A_{1}$ is said to be smooth if for every point $q \in A_{0}$, there exists a smooth map $\tilde{g}: V \rightarrow M_{1}$ defined on a neighborhood $V$ of $q$ in $M_{0}$ such that $\left.\widetilde{g}\right|_{V \cap A_{0}}=\left.g\right|_{V \cap A_{0}}$. Furthermore, a smooth map $g: A_{0} \rightarrow A_{1}$ is a diffeomorphism if it is a homeomorphism and its inverse is also smooth. When there exists a diffeomorphism between $A_{0}$ and $A_{1}$, we say that they are diffeomorphic.

Definition 2.2 Let $f_{i}: M_{i} \rightarrow N_{i}$ be smooth maps and take points $y_{i} \in N_{i}, i=0,1$. We say that the fibers over $y_{0}$ and $y_{1}$ are $C^{\infty}$ equivalent (or $C^{0}$ equivalent) if for 
some open neighborhoods $U_{i}$ of $y_{i}$ in $N_{i}$, there exist diffeomorphisms (respectively, homeomorphisms) $\tilde{\varphi}:\left(f_{0}\right)^{-1}\left(U_{0}\right) \rightarrow\left(f_{1}\right)^{-1}\left(U_{1}\right)$ and $\varphi: U_{0} \rightarrow U_{1}$ with $\varphi\left(y_{0}\right)=y_{1}$ which make the following diagram commutative:

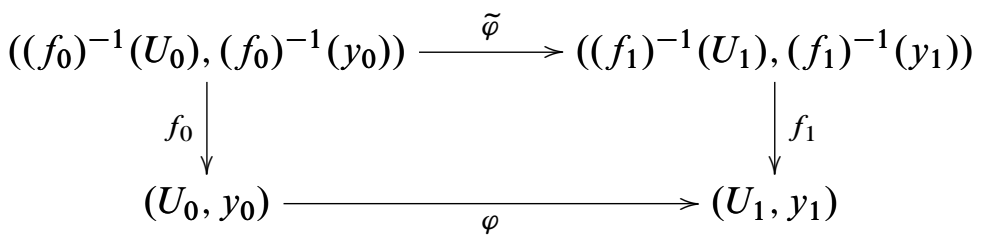

When the fibers over $y_{0}$ and $y_{1}$ are $C^{\infty}$ (or $C^{0}$ ) equivalent, we also say that the map germs $f_{0}:\left(M_{0},\left(f_{0}\right)^{-1}\left(y_{0}\right)\right) \rightarrow\left(N_{0}, y_{0}\right)$ and $f_{1}:\left(M_{1},\left(f_{1}\right)^{-1}\left(y_{1}\right)\right) \rightarrow\left(N_{1}, y_{1}\right)$ are smoothly (or topologically) right-left equivalent.

When $y \in N$ is a regular value of a smooth map $f: M \rightarrow N$ between smooth manifolds, we call $f^{-1}(y)$ a regular fiber; otherwise, a singular fiber.

Definition 2.3 Let $\mathfrak{F}$ be a $C^{0}$ equivalence class of a fiber of a proper smooth map in the sense of Definition 2.2. For a proper smooth map $f: M \rightarrow N$ between smooth manifolds, we denote by $\mathfrak{F}(f)$ the set consisting of those points of $N$ over which lies a fiber of type $\mathfrak{F}$. It is known that if the smooth map $f$ is generic enough (for example if $f$ is a Thom map, see the book by Gibson, Wirthmüller, du Plessis and Looijenga [7]), then $\mathfrak{F}(f)$ is a union of strata ${ }^{1}$ of $N$ and is a $C^{0}$ submanifold of $N$ of constant codimension (for details, see [24, Chapter 7]). Furthermore, this codimension $\kappa=\kappa(\mathfrak{F})$ does not depend on the choice of $f$ and we call it the codimension of $\mathfrak{F}$. We also say that a fiber belonging to $\mathfrak{F}$ is a codimension $\kappa$ fiber.

Let us introduce the following weaker relation for (singular) fibers.

Definition 2.4 Let $f_{i}:\left(M_{i},\left(f_{i}\right)^{-1}\left(y_{i}\right)\right) \rightarrow\left(N_{i}, y_{i}\right)$ be proper smooth map germs along fibers with $n=\operatorname{dim} M_{i}$ and $p=\operatorname{dim} N_{i}, i=0,1$, with $n \geq p$. We may assume that $N_{i}$ is the $p$-dimensional open disk Int $D^{p}$ and that $y_{i}$ is its center $0, i=0,1$. We say that the two fibers are $C^{0}$ (or $C^{\infty}$ ) equivalent modulo regular fibers if there exist $(n-p)$-dimensional closed manifolds $F_{i}, i=0,1$, such that the disjoint union of $f_{0}$ and the map germ $\pi_{0}:\left(F_{0} \times \operatorname{Int} D^{p}, F_{0} \times\{0\}\right) \rightarrow\left(\operatorname{Int} D^{p}, 0\right)$ defined by the projection to the second factor is $C^{0}$ (respectively, $C^{\infty}$ ) equivalent to the disjoint union of $f_{1}$ and the map germ $\pi_{1}:\left(F_{1} \times \operatorname{Int} D^{p}, F_{1} \times\{0\}\right) \rightarrow\left(\operatorname{Int} D^{p}, 0\right)$ defined by the projection to the second factor.

\footnotetext{
${ }^{1}$ In the case where $f$ is a Thom map, we consider the stratifications of $M$ and $N$ with respect to which $f$ satisfies certain regularity conditions. For details, see [7, Chapter I, Section 3].
} 
Note that by the very definition, any two regular fibers are $C^{\infty}$ equivalent modulo regular fibers to each other as long as their dimensions of the source and the target are the same.

For the $C^{0}$ equivalence modulo regular fibers, we use the same notation as in Definition 2.3. Then all the assertions in Definition 2.3 hold for $C^{0}$ equivalence classes modulo regular fibers as well.

The following definition is not so important in this paper. However, in order to compare it with Definition 2.6, we recall it. For details, refer to [24].

Definition 2.5 Let $\mathfrak{F}$ be a $C^{0}$ equivalence class of a fiber of a proper Thom map. Let us consider arbitrary homeomorphisms $\tilde{\varphi}$ and $\varphi$ which make the diagram

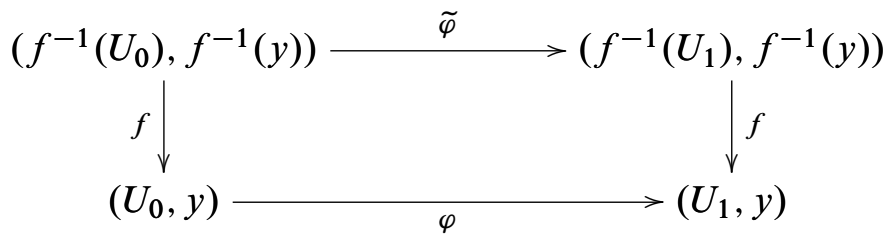

commutative, where $f$ is a proper Thom map such that the fiber over $y$ belongs to $\mathfrak{F}$, and $U_{i}$ are open neighborhoods of $y$. Note that then we have $\varphi\left(\mathfrak{F}(f) \cap U_{0}\right)=$ $\mathfrak{F}(f) \cap U_{1}$. We say that $\mathfrak{F}$ is co-orientable if $\varphi$ always preserves the local orientation of the normal bundle of $\mathfrak{F}(f)$ at $y$.

We also call any fiber belonging to a co-orientable $C^{0}$ equivalence class a co-orientable fiber.

In particular, if the codimension of $\mathfrak{F}$ coincides with the dimension of the target of $f$, then $\varphi$ above should preserve the local orientation of the target at $y$.

Note that if $\mathfrak{F}$ is co-orientable, then $\mathfrak{F}(f)$ has orientable normal bundle for every proper Thom map $f$.

The following definition plays an essential role in this paper. Compare this with Definition 2.5.

Definition 2.6 Let $\mathfrak{F}$ be a $C^{0}$ equivalence class of a fiber of a proper Thom map of an oriented manifold. We say that $\mathfrak{F}$ is achiral if there exist homeomorphisms $\tilde{\varphi}$ and 
$\varphi$ which make the diagram

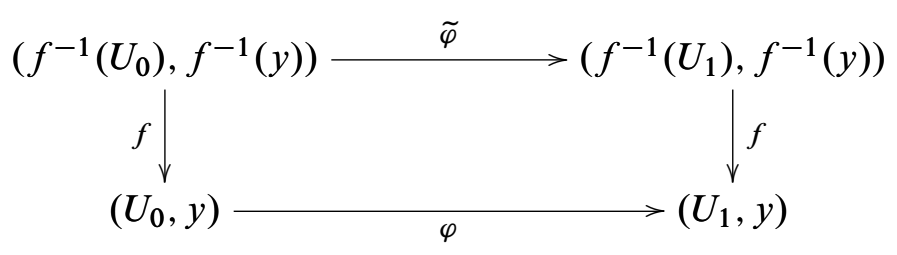

commutative such that the homeomorphism $\tilde{\varphi}$ reverses the orientation and that the homeomorphism

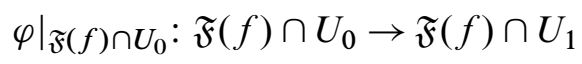

preserves the local orientation of $\mathfrak{F}(f)$ at $y$, where $f$ is a proper Thom map such that the fiber over $y$ belongs to $\mathfrak{F}$, and $U_{i}$ are open neighborhoods of $y$.

Note that if the codimension of $\mathfrak{F}$ coincides with the dimension of the target of $f$, then the condition about the homeomorphism (2-2) is redundant. Note also that the above definition does not depend on the choice of $f$ or $y$.

Moreover, we say that $\mathfrak{F}$ is chiral if it is not achiral.

We also call any fiber belonging to a chiral (respectively, achiral) $C^{0}$ equivalence class a chiral fiber (respectively, achiral fiber).

Furthermore, we have the following.

Lemma 2.7 Suppose that the codimension of $\mathfrak{F}$ is strictly smaller than the dimension of the target. Then $\mathfrak{F}$ is achiral if and only if there exist homeomorphisms $\tilde{\varphi}$ and $\varphi$ making the diagram (2-1) commutative such that the homeomorphism $\tilde{\varphi}$ preserves the orientation and that the homeomorphism (2-2) reverses the orientation.

Proof Let $f:\left(M, f^{-1}(y)\right) \rightarrow(N, y)$ be a representative of $\mathfrak{F}$, which is a proper Thom map. Let us consider the Whitney stratifications $\mathcal{M}$ and $\mathcal{N}$ of $M$ and $N$ respectively with respect to which $f$ satisfies certain regularity conditions [7, Chapter I, Section 3]. We may assume that $y$ belongs to a top dimensional stratum of $\mathfrak{F}(f)$ with respect to $\mathcal{N}$. By our hypothesis, the dimension $k$ of this stratum is strictly positive. Let $\Delta$ be a small open disk of codimension $k$ centered at $y$ in $N$ which intersects with the stratum transversely at $y$. Set $M^{\prime}=f^{-1}(\Delta)$. Note that $f^{\prime}=\left.f\right|_{M^{\prime}}: M^{\prime} \rightarrow \Delta$ is a proper Thom map.

By the second isotopy lemma (for example, see [7, Chapter II, Section 5]), we see that the map germ

$$
f:\left(M, f^{-1}(y)\right) \rightarrow(N, y)
$$


is $C^{0}$ equivalent to the map germ

$$
f^{\prime} \times \operatorname{id}_{\mathbb{R}^{k}}:\left(M^{\prime} \times \mathbb{R}^{k}, f^{\prime-1}(y) \times 0\right) \rightarrow\left(\Delta \times \mathbb{R}^{k}, y \times 0\right) .
$$

Since $k$ is positive, it is now easy to construct orientation reversing homeomorphisms $\tilde{\varphi}$ and $\varphi$ making the diagram (2-1) commutative such that the homeomorphism (2-2) reverses the orientation. Then the lemma follows immediately. This completes the proof.

We warn the reader that even if a fiber is chiral, homeomorphisms $\tilde{\varphi}$ and $\varphi$ making the diagram (2-1) commutative may not satisfy any of the following.

(1) The homeomorphism $\tilde{\varphi}$ preserves the orientation and the homeomorphism (2-2) preserves the orientation.

(2) The homeomorphism $\tilde{\varphi}$ reverses the orientation and the homeomorphism (2-2) reverses the orientation.

This is because $f^{-1}\left(U_{i}\right)$ may not be connected.

For example, a regular fiber is achiral if and only if the fiber manifold admits an orientation reversing homeomorphism. The disjoint union of an achiral fiber and an achiral regular fiber is clearly achiral. The disjoint union of a chiral fiber and an achiral regular fiber is always chiral.

In what follows, we consider only those maps of codimension -1 so that a regular fiber is always of dimension 1 . Note that every compact 1-dimensional manifold admits an orientation reversing homeomorphism. Therefore, for two fibers which are $C^{0}$ equivalent modulo regular fibers, one is chiral if and only if so is the other. Therefore, we can speak of a chiral (or achiral) $C^{0}$ equivalence class modulo regular fibers as well.

\section{Singular fibers of stable maps of 4-manifolds into 3-man- ifolds}

In this section, we consider proper $C^{\infty}$ stable maps of orientable 4-manifolds into 3-manifolds and recall the classification of their singular fibers obtained in [24].

Let $M$ and $N$ be manifolds. We say that a smooth map $f: M \rightarrow N$ is $C^{\infty}$ stable (or stable for short) if the $\mathcal{A}$-orbit of $f$ is open in the mapping space $C^{\infty}(M, N)$ with respect to the Whitney $C^{\infty}$-topology. Here, the $\mathcal{A}$-orbit of $f \in C^{\infty}(M, N)$ means the following. Let Diff $M$ (or Diff $N$ ) denote the group of all diffeomorphisms 
of the manifold $M$ (respectively, $N$ ). Then Diff $M \times \operatorname{Diff} N$ acts on $C^{\infty}(M, N)$ by $(\Phi, \Psi) \cdot f=\Psi \circ f \circ \Phi^{-1}$ for $(\Phi, \Psi) \in \operatorname{Diff} M \times \operatorname{Diff} N$ and $f \in C^{\infty}(M, N)$. Then the $\mathcal{A}$-orbit of $f \in C^{\infty}(M, N)$ means the orbit through $f$ with respect to this action. Note that a proper $C^{\infty}$ stable map is always a Thom map.

Since $(4,3)$ is a nice dimension pair in the sense of Mather [14], if $\operatorname{dim} M=4$ and $\operatorname{dim} N=3$, then the set of all $C^{\infty}$ stable maps is open and dense in $C^{\infty}(M, N)$ as long as $M$ is compact. In particular, every smooth map $M \rightarrow N$ can be approximated arbitrarily well by a $C^{\infty}$ stable map. This shows the abundance of such stable maps.

The following characterization of proper $C^{\infty}$ stable maps of 4-manifolds into 3manifolds is well-known (for example, see [24]).

Proposition 3.1 A proper smooth map $f: M \rightarrow N$ of a 4-manifold $M$ into a 3 -manifold $N$ is $C^{\infty}$ stable if and only if the following conditions are satisfied.

(i) For every $q \in M$, there exist local coordinates $(x, y, z, w)$ and $(X, Y, Z)$ around $q \in M$ and $f(q) \in N$ respectively such that one of the following holds:

$$
(X \circ f, Y \circ f, Z \circ f)= \begin{cases}(x, y, z) & q \text { a regular point } \\ \left(x, y, z^{2}+w^{2}\right) & q \text { a definite fold point } \\ \left(x, y, z^{2}-w^{2}\right) & q \text { an indefinite fold point } \\ \left(x, y, z^{3}+x z-w^{2}\right) & q \text { a cusp point } \\ \left(x, y, z^{4}+x z^{2}+y z+w^{2}\right) & q \text { a definite swallowtail point } \\ \left(x, y, z^{4}+x z^{2}+y z-w^{2}\right) & q \text { an indefinite swallowtail point }\end{cases}
$$

(ii) Set $S(f)=\left\{q \in M\right.$ : rank $\left.d f_{q}<3\right\}$, which is a regular closed 2-dimensional submanifold of $M$ under the above condition (i). Then, for every $r \in f(S(f))$, $f^{-1}(r) \cap S(f)$ consists of at most three points and the multi-germ

$$
\left(\left.f\right|_{S(f)}, f^{-1}(r) \cap S(f)\right)
$$

is smoothly right-left equivalent to one of the six multi-germs as described in Figure 1: (1) represents a single immersion germ which corresponds to a fold point, (2) and (4) represent normal crossings of two and three immersion germs, respectively, each of which corresponds to a fold point, (3) corresponds to a cusp point, (5) represents a transverse crossing of a cuspidal edge as in (3) and an immersion germ corresponding to a fold point, and (6) corresponds to a swallowtail point.

In the following, we assume that the 4-manifold $M$ is orientable. Using Proposition 3.1, the first named author obtained the following classification of singular fibers [24]. 


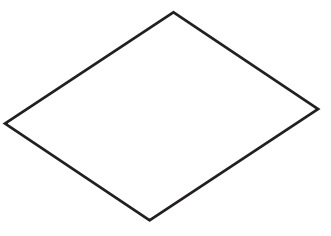

(1)

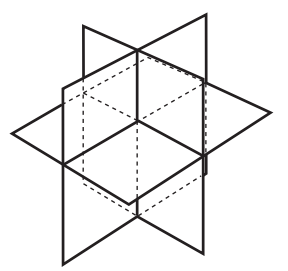

(4)

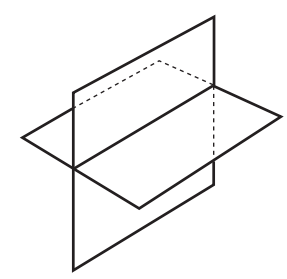

(2)

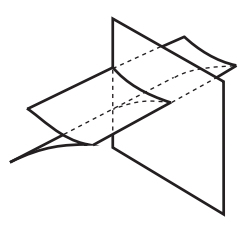

(5)

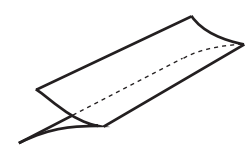

(3)

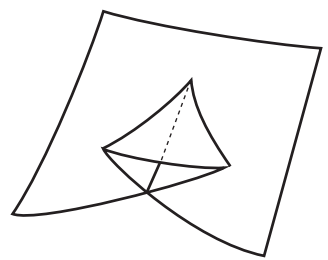

(6)

Figure 1: Multi-germs of $\left.f\right|_{S(f)}$

Theorem 3.2 Let $f: M \rightarrow N$ be a proper $C^{\infty}$ stable map of an orientable 4manifold $M$ into a 3-manifold $N$. Then, every singular fiber of $f$ is $C^{\infty}$ (and hence $C^{0}$ ) equivalent modulo regular fibers to one of the fibers as in Figure 2. Furthermore, no two fibers appearing in the list are $C^{0}$ equivalent modulo regular fibers.

Remark 3.3 In Figure 2, $\kappa$ denotes the codimension of the relevant singular fiber in the sense of Definition 2.3. Furthermore, $\mathrm{I}^{*}, \mathrm{II}^{*}$ and $\mathrm{III}^{*}$ mean the names of the corresponding singular fibers, and "/" is used only for separating the figures. Note that we have named the fibers so that each connected fiber has its own digit or letter, and a disconnected fiber has the name consisting of the digits or letters of its connected components. Hence, the number of digits or letters in the superscript coincides with the number of connected components that contain singular points.

Remark 3.4 For proper $C^{\infty}$ stable maps of 3-manifolds into the plane, a similar classification of singular fibers was obtained by Kushner, Levine and Porto [10; 12], although they did not mention explicitly the equivalence relation for their classification. Their classification was in fact based on the "diffeomorphism modulo regular fibers".

Remark 3.5 For proper $C^{\infty}$ stable maps of general (possibly nonorientable) 4manifolds into 3-manifolds, a similar classification of singular fibers was obtained by the second named author in $[28 ; 27]$. 


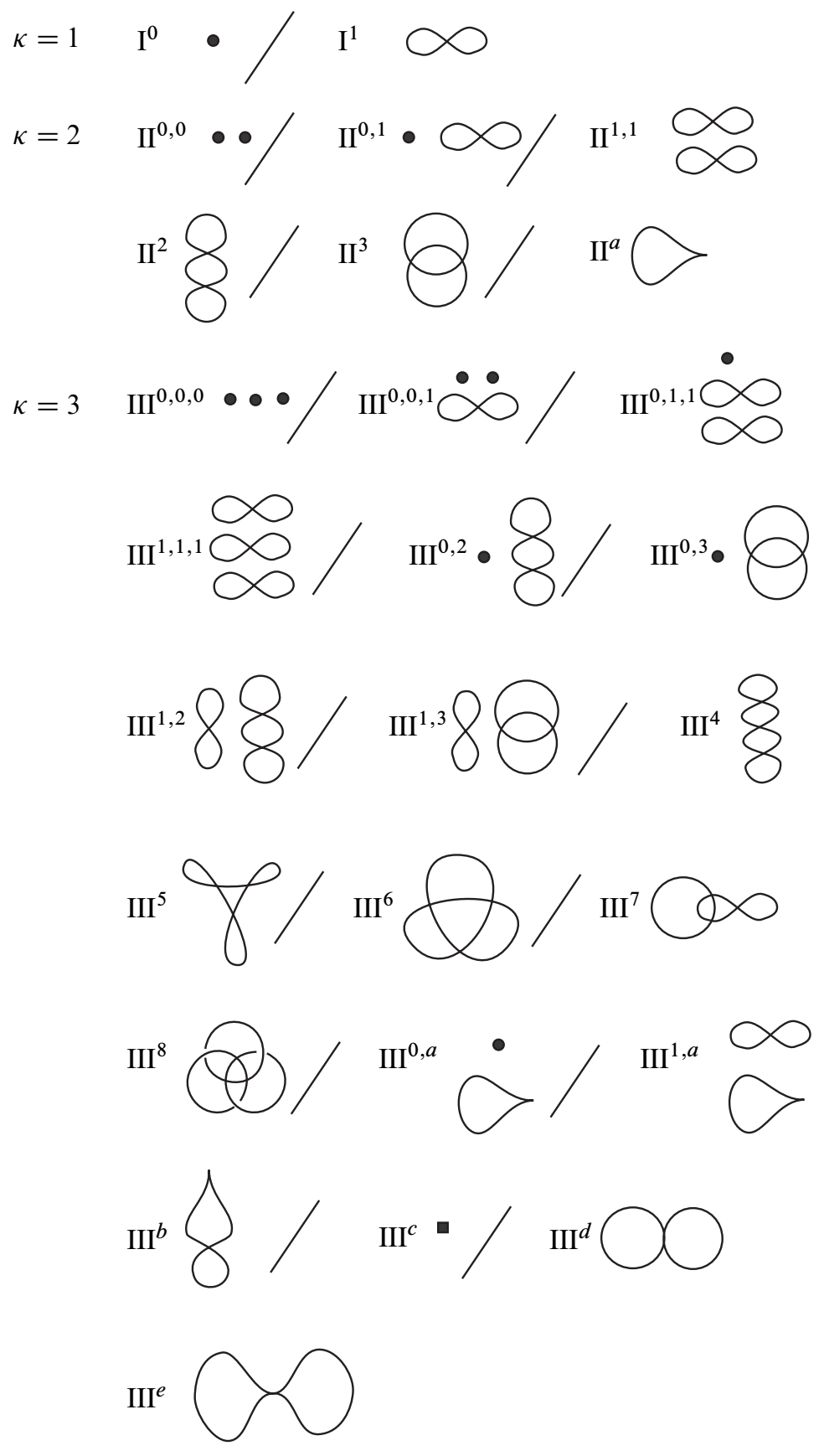

Figure 2: List of singular fibers of proper $C^{\infty}$ stable maps of orientable 4-manifolds into 3-manifolds 


\section{Singular fibers of stable maps of 5-manifolds into 4-man- ifolds}

In this section we give a characterization of $C^{\infty}$ stable maps of 5-manifolds into 4-manifolds and present the classification of singular fibers of such maps.

First note that since $(5,4)$ is a nice dimension pair in the sense of Mather [14], for a 5-manifold $M$ and a 4-manifold $N$, the set of all $C^{\infty}$ stable maps is open and dense in the mapping space $C^{\infty}(M, N)$, as long as $M$ is compact.

By using standard methods in singularity theory (for example, see the book [8] by Golubitsky and Guillemin), together with a result of Ando [1], we can prove the following characterization of stable maps of 5-manifolds into 4-manifolds.

Proposition 4.1 A proper smooth map $f: M \rightarrow N$ of a 5-manifold $M$ into a 4-manifold $N$ is $C^{\infty}$ stable if and only if the following conditions are satisfied.

(i) For every $q \in M$, there exist local coordinates $(a, b, c, x, y)$ and $(X, Y, Z, W)$ around $q \in M$ and $f(q) \in N$ respectively such that one of the following holds:

$$
\begin{aligned}
& (X \circ f, Y \circ f, Z \circ f, W \circ f)= \\
& \begin{cases}(a, b, c, x) & q \text { a regular point } \\
\left(a, b, c, x^{2}+y^{2}\right) & q \text { a definite fold point } \\
\left(a, b, c, x^{2}-y^{2}\right) & q \text { an indefinite fold point } \\
\left(a, b, c, x^{3}+a x-y^{2}\right) & q \text { a cusp point } \\
\left(a, b, c, x^{4}+a x^{2}+b x+y^{2}\right) & q \text { a definite swallowtail point } \\
\left(a, b, c, x^{4}+a x^{2}+b x-y^{2}\right) & q \text { an indefinite swallowtail point } \\
\left(a, b, c, x^{5}+a x^{3}+b x^{2}+c x-y^{2}\right) & q \text { a butterfly point } \\
\left(a, b, c, 3 x^{2} y+y^{3}+a\left(x^{2}+y^{2}\right)+b x+c y\right) & q \text { a definite } D_{4} \text { point } \\
\left(a, b, c, 3 x^{2} y-y^{3}+a\left(x^{2}+y^{2}\right)+b x+c y\right) & q \text { an indefinite } D_{4} \text { point }\end{cases}
\end{aligned}
$$

(ii) Set $S(f)=\left\{q \in M\right.$ : rank $\left.d f_{q}<4\right\}$, which is a regular closed 3-dimensional submanifold of $M$ under the above condition (i). Then, for every $r \in f(S(f))$, $f^{-1}(r) \cap S(f)$ consists of at most four points and the multi-germ

$$
\left(\left.f\right|_{S(f)}, f^{-1}(r) \cap S(f)\right)
$$

is smoothly right-left equivalent to one of the thirteen multi-germs as follows:

(1) A single immersion germ which corresponds to a fold point 
(2) A normal crossing of two immersion germs, each of which corresponds to a fold point

(3) A cuspidal edge which corresponds to a single cusp point

(4) A normal crossing of three immersion germs, each of which corresponds to a fold point

(5) A transverse crossing of a cuspidal edge and an immersion germ corresponding to a fold point

(6) A map germ corresponding to a swallowtail point

(7) A normal crossing of four immersion germs, each of which corresponds to a fold point

(8) A transverse crossing of a cuspidal edge and a normal crossing of two immersion germs which correspond to fold points

(9) A transverse crossing of two cuspidal edges

(10) A transverse crossing of a swallowtail germ and an immersion germ corresponding to a fold point

(11) A map germ corresponding to a butterfly point

(12) A map germ corresponding to a definite $D_{4}$ point

(13) A map germ corresponding to an indefinite $D_{4}$ point

We call a $D_{4}$ point a $\Sigma^{2,2,0}$ point as well.

Remark 4.2 The normal forms for $D_{4}$ points are slightly different from the usual ones (see, for example, the article [1] by Ando). We have chosen them so that at an indefinite $D_{4}$ point, $f$ can be represented as

$$
(a, \eta, \zeta) \mapsto\left(a, \eta, \Im\left(\zeta^{3}\right)+\Re(\bar{\eta} \zeta)+a|\zeta|^{2}\right)
$$

by using complex numbers, where $i=\sqrt{-1}, \eta=b+i c, \zeta=x+i y$, $\Im$ means the imaginary part, and $\mathfrak{R}$ means the real part.

Set $\tau=\exp (2 \pi i / 3)$. Then with respect to the chosen coordinates, we have

$$
f \circ \tilde{\varphi}_{\tau}=\varphi_{\tau} \circ f,
$$

where $\tilde{\varphi}_{\tau}$ and $\varphi_{\tau}$ are orientation preserving diffeomorphisms defined by

$$
\begin{aligned}
\tilde{\varphi}_{\tau}(a, \eta, \zeta) & =(a, \tau \eta, \tau \zeta), \quad \text { and } \\
\varphi_{\tau}(X, Y+i Z, W) & =(X, \tau(Y+i Z), W)
\end{aligned}
$$


respectively. This shows that an indefinite $D_{4}$ point (or a local fiber through an indefinite $D_{4}$ point) has a (orientation preserving) symmetry of order 3 .

Set $\tau^{\prime}=\exp (\pi i / 3)$ so that we have $\tau^{\prime 2}=\tau$. Then we have

$$
f \circ \tilde{\varphi}_{\tau^{\prime}}=\varphi_{\tau^{\prime}} \circ f
$$

where $\tilde{\varphi}_{\tau^{\prime}}$ and $\varphi_{\tau^{\prime}}$ are diffeomorphisms defined by

$$
\begin{aligned}
\tilde{\varphi}_{\tau^{\prime}}(a, \eta, \zeta) & =\left(-a,-\tau^{\prime} \eta, \tau^{\prime} \zeta\right), \quad \text { and } \\
\varphi_{\tau}(X, Y+i Z, W) & =\left(-X,-\tau^{\prime}(Y+i Z),-W\right)
\end{aligned}
$$

respectively. Note that $\tilde{\varphi}_{\tau^{\prime}}$ is orientation reversing while $\varphi_{\tau^{\prime}}$ is orientation preserving. This shows that an indefinite $D_{4}$ point (or a local fiber through an indefinite $D_{4}$ point) has a symmetry of order 6 and that the generator reverses the "local orientation" of the fiber. In fact, we have $\tilde{\varphi}_{\tau}=\widetilde{\varphi}_{\tau^{\prime}}^{2}$ and $\varphi_{\tau}=\varphi_{\tau^{\prime}}^{2}$.

Let us recall the following definition (for details, see [24, Chapter 8]).

Definition 4.3 Let $f: M \rightarrow N$ be a proper smooth map between manifolds. Then we call the proper smooth map

$$
f \times \operatorname{id}_{\mathbb{R}}: M \times \mathbb{R} \rightarrow N \times \mathbb{R}
$$

the suspension of $f$. Furthermore, to the fiber of $f$ over a point $y \in N$, we can associate the fiber of $f \times \operatorname{id}_{\mathbb{R}}$ over $y \times\{0\}$. We say that the latter fiber is obtained from the original fiber by the suspension. Note that a fiber and its suspension are diffeomorphic to each other in the sense of Definition 2.1.

Note that the map germs (1)-(6) in Proposition 4.1 correspond to the suspensions of the map germs in Figure 1. The map germs (11)-(13) are as described in Figures 3-5 respectively, where in order to draw 3-dimensional objects in a 4-dimensional space, we have depicted three "sections" by 3-dimensional spaces for each object.
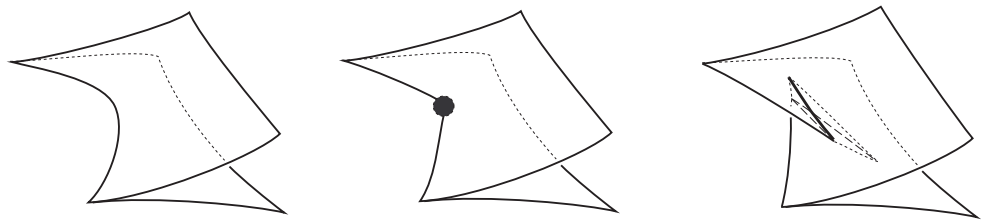

Figure 3: Map germ corresponding to a butterfly point 

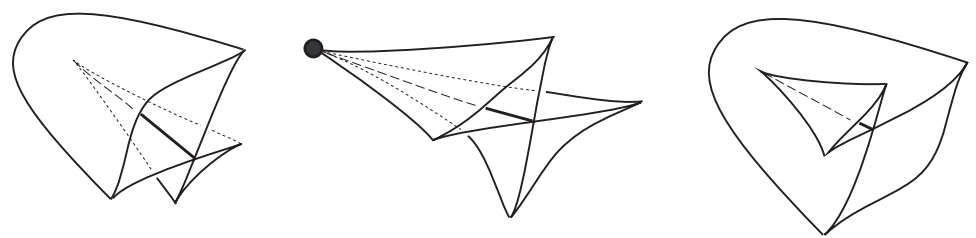

Figure 4: Map germ corresponding to a definite $D_{4}$ point
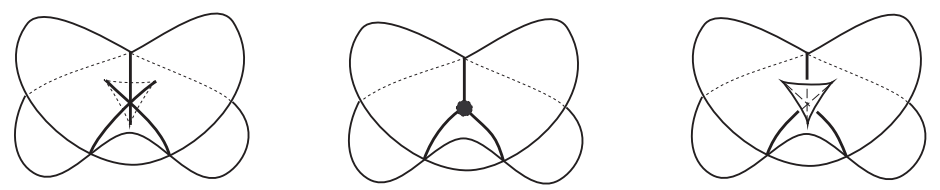

Figure 5: Map germ corresponding to an indefinite $D_{4}$ point

Let $q$ be a singular point of a proper $C^{\infty}$ stable map $f: M \rightarrow N$ of a 5-manifold $M$ into a 4-manifold $N$. Then, using the above local normal forms, we can easily describe the diffeomorphism type of a neighborhood of $q$ in $f^{-1}(f(q))$. More precisely, we easily get the following local characterizations of singular fibers.

Lemma 4.4 Every singular point $q$ of a proper $C^{\infty}$ stable map $f: M \rightarrow N$ of a 5-manifold $M$ into a 4-manifold $N$ has one of the following neighborhoods in its corresponding singular fiber (see Figure 6):

(1) isolated point diffeomorphic to $\left\{(x, y) \in \mathbb{R}^{2}: x^{2}+y^{2}=0\right\}$, if $q$ is a definite fold point,

(2) union of two transverse arcs diffeomorphic to $\left\{(x, y) \in \mathbb{R}^{2}: x^{2}-y^{2}=0\right\}$, if $q$ is an indefinite fold point,

(3) (2,3)-cuspidal arc diffeomorphic to $\left\{(x, y) \in \mathbb{R}^{2}: x^{3}-y^{2}=0\right\}$, if $q$ is a cusp point,

(4) isolated point diffeomorphic to $\left\{(x, y) \in \mathbb{R}^{2}: x^{4}+y^{2}=0\right\}$, if $q$ is a definite swallowtail point,

(5) union of two tangent arcs diffeomorphic to $\left\{(x, y) \in \mathbb{R}^{2}: x^{4}-y^{2}=0\right\}$, if $q$ is an indefinite swallowtail point,

(6) $(2,5)$-cuspidal arc diffeomorphic to $\left\{(x, y) \in \mathbb{R}^{2}: x^{5}-y^{2}=0\right\}$, if $q$ is a butterfly point,

(7) non-disjoint union of an arc and a point diffeomorphic to $\left\{(x, y) \in \mathbb{R}^{2}: 3 x^{2} y+\right.$ $\left.y^{3}=0\right\}$, if $q$ is a definite $D_{4}$ point, 
(8) union of three arcs meeting at a point with distinct tangents diffeomorphic to $\left\{(x, y) \in \mathbb{R}^{2}: 3 x^{2} y-y^{3}=0\right\}$, if $q$ is an indefinite $D_{4}$ point.

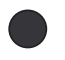

(1)

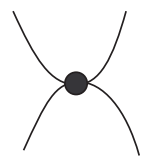

(5)

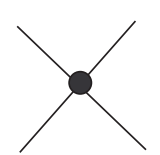

(2)

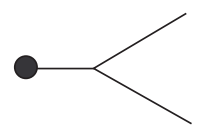

(6)

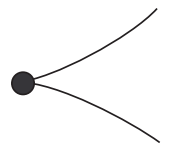

(3)

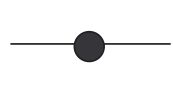

(7)
(4)

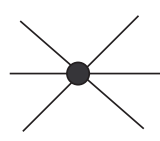

(8)

Figure 6: Neighborhood of a singular point in a singular fiber

We note that in Figure 6, both the black dot (1) and the black square (4) represent an isolated point; however, we use distinct symbols in order to distinguish them. We also use the symbols as in Figure 6 (3) and (6) in order to distinguish a $(2,3)$-cusp from a $(2,5)$-cusp. Furthermore, we put a dot on the arc as in Figure 6 (7) in order to distinguish it from a regular fiber.

Then by an argument similar to that in [24, Chapter 3], we can prove the following, whose proof is left to the reader.

Theorem 4.5 Let $f: M \rightarrow N$ be a proper $C^{\infty}$ stable map of an orientable 5manifold $M$ into a 4-manifold $N$. Then, every singular fiber of $f$ is $C^{0}$ equivalent modulo regular fibers to one of the fibers as follows:

(1) The suspension of a fiber appearing in Theorem 3.2

(2) One of the disconnected fibers

$$
\begin{aligned}
& \mathrm{IV}^{0,0,0,0}, \mathrm{IV}^{0,0,0,1}, \mathrm{IV}^{0,0,1,1}, \mathrm{IV}^{0,1,1,1}, \mathrm{IV}^{1,1,1,1}, \mathrm{IV}^{0,0,2}, \mathrm{IV}^{0,1,2}, \mathrm{IV}^{1,1,2}, \\
& \mathrm{IV}^{0,0,3}, \mathrm{IV}^{0,1,3}, \mathrm{IV}^{1,1,3}, \mathrm{IV}^{0,4}, \mathrm{IV}^{0,5}, \mathrm{IV}^{0,6}, \mathrm{IV}^{0,7}, \mathrm{IV}^{0,8}, \mathrm{IV}^{1,4}, \mathrm{IV}^{1,5}, \\
& \mathrm{IV}^{1,6}, \mathrm{IV}^{1,7}, \mathrm{IV}^{1,8}, \mathrm{IV}^{2,2}, \mathrm{IV}^{2,3}, \mathrm{IV}^{3,3}, \mathrm{IV}^{0,0, a}, \mathrm{IV}^{0,1, a}, \mathrm{IV}^{1,1, a}, \mathrm{IV}^{0, b}, \\
& \mathrm{IV}^{1, b}, \mathrm{IV}^{2, a}, \mathrm{IV}^{3, a}, \mathrm{IV}^{a, a}, \mathrm{IV}^{0, c}, \mathrm{IV}^{0, d}, \mathrm{IV}^{0, e}, \mathrm{IV}^{1, c}, \mathrm{IV}^{1, d}, \mathrm{IV}^{1, e}
\end{aligned}
$$

(3) One of the connected fibers depicted in Figure 7

Furthermore, no two fibers appearing in the list (1)-(3) above are $C^{0}$ equivalent modulo regular fibers. 

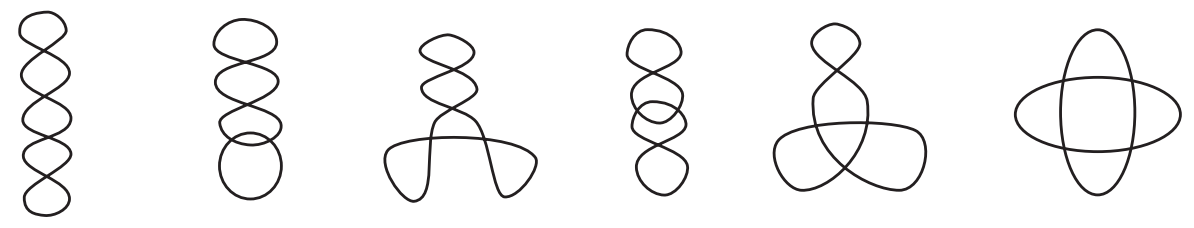

$\mathrm{IV}^{9}$

$\mathrm{IV}^{10}$

$\mathrm{IV}^{11}$

$\mathrm{IV}^{12}$

$\mathrm{IV}^{13}$

$\mathrm{IV}^{14}$
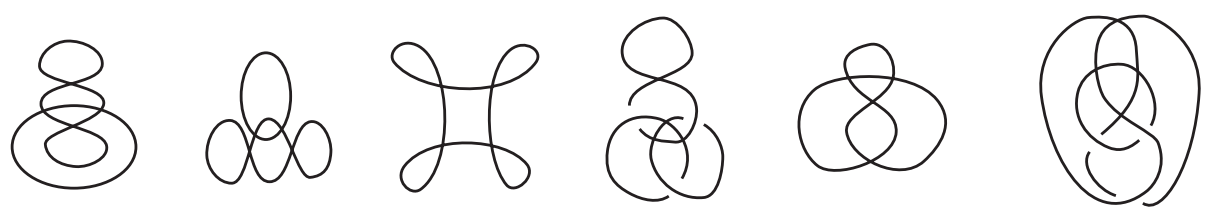

IV $^{15}$

$\mathrm{IV}^{16}$

$\mathrm{IV}^{17}$

$\mathrm{IV}^{18}$

$\mathrm{IV}^{19}$

$\mathrm{IV}^{20}$

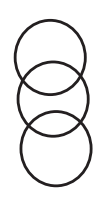

$\mathrm{IV}^{21}$
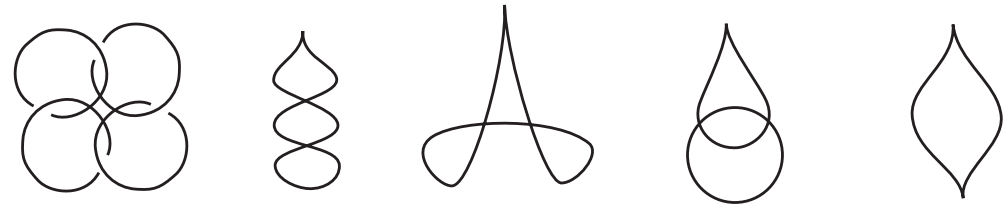

$\mathrm{IV}^{22}$

$\mathrm{IV}^{f}$

$\mathrm{IV}^{g}$

$\mathrm{IV}^{h}$

$\mathrm{IV}^{i}$

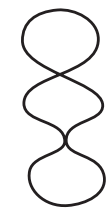

$\mathrm{IV}^{j}$

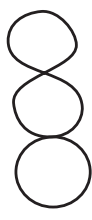

$\mathrm{IV}^{k}$

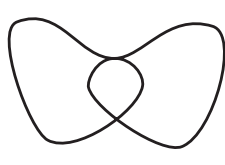

$\mathrm{IV}^{l}$

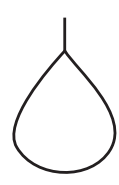

$\mathrm{IV}^{m}$

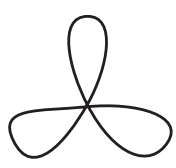

$\mathrm{IV}^{n}$

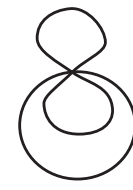

$\mathrm{IV}^{o}$
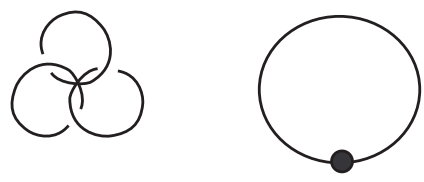

$\mathrm{IV}^{p}$

$\mathrm{IV}^{q}$

Figure 7: List of codimension 4 connected singular fibers of proper $C^{\infty}$ stable maps of orientable 5-manifolds into 4-manifolds 
For the fibers in Theorem 4.5 (1), we use the same names as those of the corresponding fibers in Theorem 3.2. Note that the names of the fibers are consistent with the convention mentioned in Remark 3.3. Therefore, the figure corresponding to each fiber listed in Theorem 4.5 (2) can be obtained by taking the disjoint union of the fibers in Figure 2 corresponding to the digits or letters appearing in the superscript. For example, the figure for the fiber $\mathrm{IV}^{0,0,0,1}$ consists of three dots and a "figure 8 ".

In Figure 7, we did not use " /" as in Figure 2, since the depicted fibers are all connected and are easy to recognize.

Note also that the codimensions of the fibers in Theorem 4.5 (1) coincide with those of the corresponding fibers in Theorem 3.2. Furthermore, the fibers in Theorem 4.5 (2) and (3) all have codimension 4.

Remark 4.6 The result of Theorem 4.5 holds for the classification up to $C^{\infty}$ equivalence as well. As a consequence, we see that two fibers are $C^{0}$ equivalent if and only if they are $C^{\infty}$ equivalent (for related results, refer to [24, Chapter 3]). This should be compared with a result of Damon [4] about stable map germs in nice dimensions.

\section{Chiral singular fibers and their signs}

In this section we determine those singular fibers of proper stable maps of oriented 4 -manifolds into 3-manifolds which are chiral. We also define a sign $(= \pm 1)$ for each chiral singular fiber of codimension 3 .

Let us first consider a fiber of type $\mathrm{III}^{8}$. Let $f:\left(M, f^{-1}(y)\right) \rightarrow(N, y)$ be a map germ representing the fiber of type III $^{8}$ with $f^{-1}(y)$ being connected, where $M$ is an orientable 4-manifold and $N$ is a 3-manifold. We assume that $M$ is oriented. Let us denote the three singular points of $f$ contained in $f^{-1}(y)$ by $q_{1}, q_{2}$ and $q_{3}$.

Let us fix an orientation of a neighborhood of $y$ in $N$. Then for every regular point $q \in f^{-1}(y)$, we can define the local orientation of the fiber near $q$ by the "fiber first" convention; that is, we give the orientation to the fiber at $q$ so that the ordered 4-tuple $\left\langle v, v_{1}, v_{2}, v_{3}\right\rangle$ of tangent vectors at $q$ gives the orientation of $M$, where $v$ is a tangent vector of the fiber at $q$ which corresponds to its orientation, and $v_{1}, v_{2}$ and $v_{3}$ are tangent vectors of $M$ at $q$ such that the ordered 3-tuple $\left\langle d f_{q}\left(v_{1}\right), d f_{q}\left(v_{2}\right), d f_{q}\left(v_{3}\right)\right\rangle$ corresponds to the local orientation of $N$ at $y$. Note that the set of regular points in $f^{-1}(y)$ consists of six open arcs and each of them gets its orientation by the above rule. 


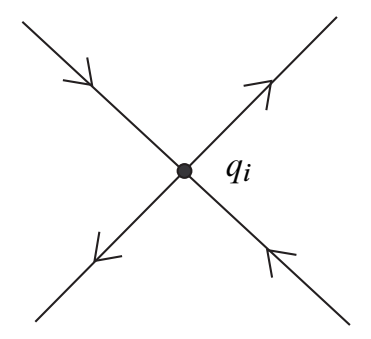

Figure 8: Orientations of the four arcs incident to a singular point

Each singular point $q_{i}$ is incident to four open arcs. We see easily that their orientations should be as depicted in Figure 8 by considering the orientations induced on the nearby fibers.

For each pair $\left(q_{i}, q_{j}\right), i \neq j$, of singular points, we have exactly two open arcs of $f^{-1}(y)$ which connect $q_{i}$ and $q_{j}$. Furthermore, the orientations of the two open arcs coincide with each other in the sense that one of the two arcs goes from $q_{i}$ to $q_{j}$ if and only if so does the other one. Then we see that the orientations on the six open arcs define a cyclic order of the three singular points $q_{1}, q_{2}$ and $q_{3}$ (see Figure 9). By renaming the three singular points if necessary, we may assume that this cyclic order is given by $\left\langle q_{1}, q_{2}, q_{3}\right\rangle$.

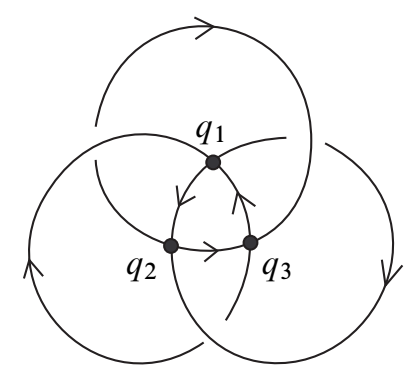

Figure 9: Cyclic order of the three singular points

Let $D_{i}$ be a sufficiently small open disk neighborhood of $q_{i}$ in $S(f)$. Since the multi-germ $\left(\left.f\right|_{S(f)}, f^{-1}(y) \cap S(f)\right)$ corresponds to the triple point as depicted in Figure 1 (4), the images $f\left(D_{1}\right), f\left(D_{2}\right)$ and $f\left(D_{3}\right)$ are open 2-disks in $N$ in general position forming a triple point at $y$. They divide a neighborhood of $y$ in $N$ into eight octants. For each octant $\omega$, take a point in it and count the number of connected components of the regular fiber over the point. It should be equal either to 1 or to 2 
and it does not depend on the choice of the point (for details, see [24, Figure 3.6]). When it is equal to $k(=1,2)$, we call $\omega$ a $k$-octant.

Choose a 1 -octant $\omega$. Let $w_{i}$ be a normal vector to $f\left(D_{i}\right)$ in $N$ pointing toward $\omega$ at a point incident to that octant, $i=1,2,3$ (see Figure 10).

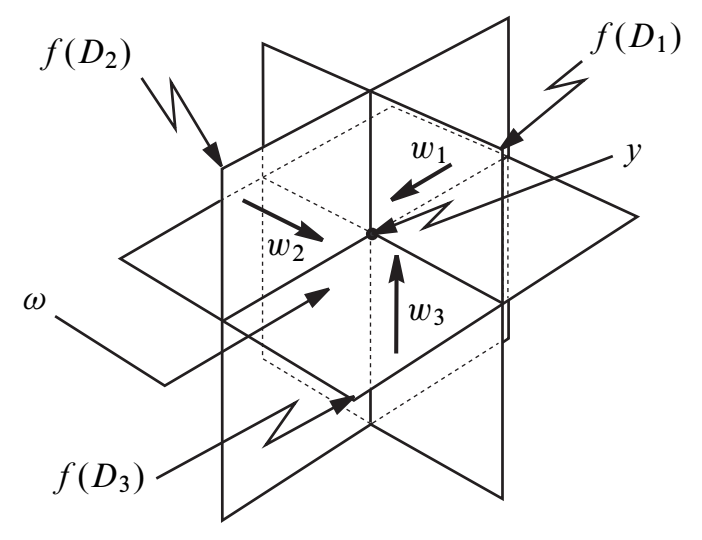

Figure 10: Vectors $w_{i}$ normal to $f\left(D_{i}\right)$ pointing toward $\omega$

We may identify a neighborhood of $y$ in $N$ with $\mathbb{R}^{3}$. Then the local orientation at $y$ corresponding to the ordered 3-tuple of vectors $\left\langle w_{1}, w_{2}, w_{3}\right\rangle$ depends only on the cyclic order of the three open disks $f\left(D_{1}\right), f\left(D_{2}\right)$ and $f\left(D_{3}\right)$ and is well-defined, once a 1 -octant is chosen. Then we say that the fiber $f^{-1}(y)$ is positive if the orientation corresponding to $\left\langle w_{1}, w_{2}, w_{3}\right\rangle$ coincides with the local orientation of $N$ at $y$ which we chose at the beginning; otherwise, negative. We define the sign of the fiber to be +1 (or -1 ) if it is positive (respectively, negative).

Lemma 5.1 The above definition does not depend on the choices of the following data, and the sign of a $\mathrm{III}^{8}$ type fiber is well-defined as long as the source 4-manifold is oriented:

(1) the 1-octant $\omega$,

(2) the local orientation of $N$ at $y$.

Proof (1) It is easy to see that any two adjacent octants have distinct numbers of connected components of their associated regular fibers; that is, a 1-octant is adjacent to 2-octants, but never to another 1-octant, and vice versa. Therefore, in order to move from the chosen 1-octant to another 1-octant, one has to cross the open disks $f\left(D_{1}\right), f\left(D_{2}\right)$ and $f\left(D_{3}\right)$ even number of times. Every time one crosses an open disk, 
the associated normal vector corresponding to that open disk changes the direction, while the other two vectors remain parallel. Therefore, after crossing the open disks even number of times, we get the same orientation determined by the associated ordered normal vectors.

(2) If we reverse the local orientation of $N$ at $y$, then the regular parts of fibers get opposite orientations. Therefore, in the above definition, the cyclic order of the three singular points is reversed. Hence, the resulting local orientation at $y$ determined by the three normal vectors is also reversed. Thus, the sign of the fiber is well-defined.

For a fiber which is a disjoint union of a III $^{8}$ type fiber and a finite number of copies of a fiber of the trivial circle bundle (that is, for a fiber equivalent to a $\mathrm{III}^{8}$ type fiber modulo regular fibers), we say that it is positive (respectively, negative) if the III $^{8}$-fiber component is positive (respectively, negative). We define the sign of such a fiber to be +1 (or -1 ) if it is positive (respectively, negative).

Remark 5.2 It should be noted that if we reverse the orientation of the source 4manifold, then the sign of a $\mathrm{III}^{8}$ type fiber necessarily changes.

Corollary 5.3 A fiber equivalent to a III $^{8}$ type fiber modulo regular fibers is always chiral.

Proof If it is achiral, then a representative of a III $^{8}$ type singular fiber and its copy with the orientation of the source 4 -manifold being reversed are $C^{0}$ equivalent with respect to an orientation preserving homeomorphism between the sources (that is, with respect to a homeomorphism $\tilde{\varphi}$ as in the diagram (2-1)). Let us take local orientations at the target points so that the homeomorphism between the target manifolds (that is, the homeomorphism $\varphi$ in the diagram (2-1)) preserves the orientation. Then by our definition of the sign, we see that the two III $^{8}$ type fibers should have the same sign, which is a contradiction in view of Remark 5.2. Therefore, the desired conclusion follows. This completes the proof.

Let us now consider the other singular fibers appearing in Theorem 3.2. By using similar arguments, we can determine the chiral singular fibers among the list. More precisely, we have the following.

Proposition 5.4 A singular fiber of a proper $C^{\infty}$ stable map of an oriented 4-manifold into a 3-manifold is chiral if and only if it contains a fiber of type $\mathrm{III}^{5}, \mathrm{III}^{7}$ or $\mathrm{III}^{8}$. 
Proof For fibers of types $\mathrm{III}^{5}$ and $\mathrm{III}^{7}$, we can define their signs as for a $\mathrm{III}^{8}$ type fiber. Therefore, they are chiral. Details are left to the reader.

For the other fibers, we can find homeomorphisms $\tilde{\varphi}$ and $\varphi$ as in Definition 2.6. For example, let us consider a $\mathrm{II}^{2}$ type fiber. Let $f:\left(M, f^{-1}(y)\right) \rightarrow(N, y)$ be a proper smooth map germ representing a fiber of type $\mathrm{II}^{2}$, and let $q_{1}$ and $q_{2}$ be the two singular points contained in $f^{-1}(y)$, both of which are indefinite fold points. We fix orientations of $M$ and $N$ near $f^{-1}(y)$ and $y$ respectively. Then the regular part of $f^{-1}(y)$ is naturally oriented by the "fiber first" convention.

It is easy to show that the involution of $f^{-1}(y)$ as in Figure 11 reverses the orientation of the regular part of $f^{-1}(y)$. Note that this involution fixes the two singular points $q_{1}$ and $q_{2}$ pointwise.

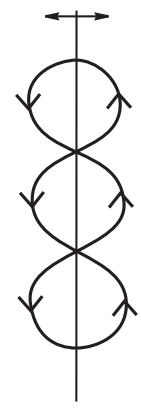

Figure 11: Orientation-reversing involution of a $\mathrm{II}^{2}$ type fiber

By Section 3, there exist coordinates $\left(x_{i}, y_{i}, z_{i}, w_{i}\right)$ and $(X, Y, Z)$ around $q_{i}, i=1,2$, and $f\left(q_{i}\right)=y$ respectively such that $f$ is given by

$$
\left(x_{1}, y_{1}, z_{1}, w_{1}\right) \mapsto\left(x_{1}, y_{1}, z_{1}^{2}-w_{1}^{2}\right)
$$

around $q_{1}$, and by

$$
\left(x_{2}, y_{2}, z_{2}, w_{2}\right) \mapsto\left(x_{2}, z_{2}^{2}-w_{2}^{2}, y_{2}\right)
$$

around $q_{2}$ with respect to these coordinates. Then we may assume that the above involution is consistent with the involutions defined by

$$
\left(x_{1}, y_{1}, z_{1}, w_{1}\right) \mapsto\left(x_{1}, y_{1},-z_{1}, w_{1}\right)
$$

around $q_{1}$ and by

$$
\left(x_{2}, y_{2}, z_{2}, w_{2}\right) \mapsto\left(x_{2}, y_{2},-z_{2}, z_{2}\right)
$$

around $q_{2}$. Then we can extend this involution of a neighborhood of $\left\{q_{1}, q_{2}\right\}$ to a self-diffeomorphism $\tilde{\varphi}$ of $f^{-1}(U)$ for a sufficiently small open disk neighborhood $U$ 
of $y$ in $N$ so that the diagram

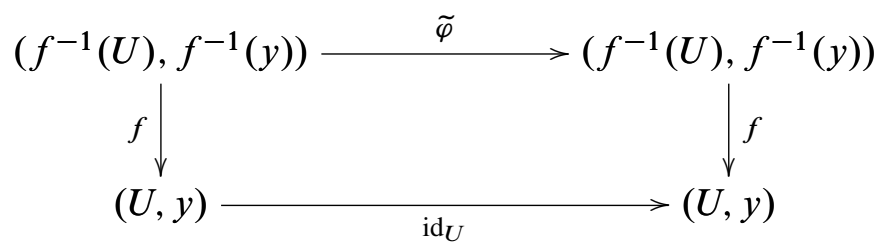

is commutative, by using the relative version of Ehresmann's fibration theorem (see Ehresmann [5], Lamotke [11, Section 3], Bröcker-Jänich [2, Section 8.12], or the book [24, Section 1] by the first named author), where $\operatorname{id}_{U}$ denotes the identity map of $U$. Note that the diffeomorphism $\tilde{\varphi}$ thus constructed is orientation reversing. Hence, the fiber $f^{-1}(y)$ is achiral according to Definition 2.6.

We can use similar arguments for the other fibers to show that they are achiral. Details are left to the reader.

Let us now state the main theorem of this paper. For a closed oriented 4-manifold, we denote by $\sigma(M)$ the signature of $M$. Furthermore, for a $C^{\infty}$ stable map $f: M \rightarrow N$ into a 3 -manifold $N$, we denote by $\left\|\mathrm{III}^{8}(f)\right\|$ the algebraic number of III $^{8}$ type fibers of $f$; that is, it is the sum of the signs over all fibers of $f$ equivalent to a III $^{8}$ type fiber modulo regular fibers.

Theorem 5.5 Let $M$ be a closed oriented 4-manifold and $N$ a 3-manifold. Then, for any $C^{\infty}$ stable map $f: M \rightarrow N$, we have

$$
\sigma(M)=\left\|\mathrm{III}^{8}(f)\right\| \in \mathbb{Z} .
$$

The proof of Theorem 5.5 will be given in Section 7.

Since for an oriented 4-manifold, the signature and the Euler characteristic have the same parity, we immediately obtain the following, which was obtained in [24].

Corollary 5.6 Let $M$ be a closed orientable 4-manifold and $N$ a 3-manifold. Then, for any $C^{\infty}$ stable map $f: M \rightarrow N$, the number of fibers of $f$ equivalent to a III $^{8}$ type fiber modulo regular fibers has the same parity as the Euler characteristic of $M$.

Note that in the proof of our main theorem, we do not use the above corollary. In other words, our proof gives a new proof for the above modulo two Euler characteristic formula. 


\section{Cobordism invariance of the algebraic number of III $^{8}$ type fibers}

In order to prove Theorem 5.5, let us first show that the algebraic number of III $^{8}$ type fibers is an oriented cobordism invariant of the source 4-manifold.

Let us begin by a list of chiral singular fibers of proper $C^{\infty}$ stable maps of 5-manifolds into 4-manifolds. We can prove the following proposition by an argument similar to that in the previous section.

Proposition 6.1 A singular fiber of a proper $C^{\infty}$ stable map of an oriented 5-manifold into a 4-manifold is chiral if and only if it is $C^{0}$ equivalent modulo regular fibers to a fiber of type $\mathrm{III}^{5}, \mathrm{III}^{7}, \mathrm{III}^{8}, \mathrm{IV}^{0,5}, \mathrm{IV}^{0,7}, \mathrm{IV}^{0,8}, \mathrm{IV}^{1,5}, \mathrm{IV}^{1,7}, \mathrm{IV}^{1,8}, \mathrm{IV}^{10}, \mathrm{IV}^{11}$, $\mathrm{IV}^{12}, \mathrm{IV}^{13}, \mathrm{IV}^{18}, \mathrm{IV}^{g}, \mathrm{IV}^{h}$, or $\mathrm{IV}^{k}$.

For example, in order to show that the fibers of types $\mathrm{IV}^{o}, \mathrm{IV}^{p}$ and $\mathrm{IV}^{q}$ are achiral, we can use the symmetry of order 6 of an indefinite $D_{4}$ point as in Remark 4.2. The proof of Proposition 6.1 is left to the reader.

Note that for each chiral singular fiber of codimension 4 , we can define its sign $(= \pm 1)$, as long as the source 5-manifold is oriented. In what follows, we fix such a definition of a sign for each chiral singular fiber of codimension 4 once and for all, although we do not mention it explicitly.

Let $\mathfrak{F}$ be a $C^{0}$ equivalence class modulo regular fibers. For a proper $C^{\infty}$ stable map $f: M \rightarrow N$ of an oriented 5-manifold $M$ into a 4-manifold $N$, we denote by $\mathfrak{F}(f)$ the set of all $y \in N$ over which lies a fiber of type $\mathfrak{F}$. Note that $\mathfrak{F}(f)$ is a regular $C^{\infty}$ submanifold of $N$ of codimension $\kappa(\mathfrak{F})$, where $\kappa(\mathfrak{F})$ denotes the codimension of the $C^{0}$ equivalence class modulo regular fibers $\mathfrak{F}$.

In general, if $\mathfrak{F}$ is chiral, then $\mathfrak{F}(f)$ is orientable. For $\mathfrak{F}=\mathrm{III}^{8}$, we introduce the orientation on $\operatorname{III}^{8}(f)$ as follows.

Take a point $y \in \operatorname{III}^{8}(f)$. Note that the singular value set $f(S(f))$ near $y$ consists of three codimension 1 "sheets" meeting along $\operatorname{III}^{8}(f)$ in general position. Let $D_{y}$ be a small open 3-disk centered at $y$ in $N$ which intersects III $^{8}(f)$ transversely exactly at $y$ and is transverse to the three sheets of $f(S(f))$. Put $M^{\prime}=f^{-1}\left(D_{y}\right)$, which is a smooth 4-dimensional submanifold of $M$ with trivial normal bundle and hence is orientable. Let us consider the proper smooth map

$$
h=\left.f\right|_{f^{-1}\left(D_{y}\right)}: M^{\prime} \rightarrow D_{y},
$$


which is a $C^{\infty}$ stable map by virtue of Proposition 3.1. Note that the fiber of $h$ over $y$ is of type $\mathrm{III}^{8}$. Let $M^{\prime \prime}$ be the component of $M^{\prime}$ containing the $\mathrm{III}^{8}$ type fiber.

Let $\mathcal{O}_{M^{\prime \prime}}$ be the orientation of $M^{\prime \prime}$ with respect to which the III $^{8}$ type fiber is positive. Then let $\mathcal{O}_{v}$ be the orientation of the normal bundle $v$ to $M^{\prime \prime}$ in $M$ such that $\mathcal{O}_{v} \oplus \mathcal{O}_{M}{ }^{\prime \prime}$ is consistent with the orientation of the 5-manifold $M$. By the differential $d f: T M \rightarrow$ $T N$ at a point in $M^{\prime \prime}, \mathcal{O}_{v}$ corresponds to a normal direction to $D_{y}$ in $N$ at $y$. Now we orient $\operatorname{III}^{8}(f)$ at $y$ so that this direction is consistent with the orientation of $\operatorname{III}^{8}(f)$. It is easy to see that this orientation varies continuously with respect to $y \in \mathrm{III}^{8}(f)$ and hence defines an orientation on $\operatorname{III}^{8}(f)$.

Now let $\mathfrak{F}$ be the $C^{0}$ equivalence class modulo regular fibers of one of the codimension 4 fibers appearing in Proposition 6.1; that is, $\mathfrak{F}$ is a chiral singular fiber of codimension 4. Note that $\mathfrak{F}(f)$ is a discrete set in $N$. Take a point $y \in \mathfrak{F}(f)$ and a sufficiently small open disk neighborhood $\Delta_{y}$ of $y$ in $N$. We orient the source 5-manifold so that the fiber over $y$ gets the sign +1 . Then $\Delta_{y} \cap \mathrm{III}^{8}(f)$ consists of several oriented arcs which have a common end point at $y$. Let us define the incidence coefficient $\left[\mathrm{III}^{8}: \mathfrak{F}\right] \in \mathbb{Z}$ to be the number of arcs coming into $y$ minus the number of arcs going out of $y$. Note that this does not depend on the point $y$ nor on the map $f$.

Remark 6.2 Let $\mathfrak{F}$ be the $C^{0}$ equivalence class modulo regular fibers of a codimension 4 achiral singular fiber. Then we can define the incidence coefficient $\left[\mathrm{III}^{8}: \mathfrak{F}\right] \in \mathbb{Z}$ in exactly the same manner as above. However, this should always vanish, since the homeomorphism $\varphi$ as in (2-1) reverses the orientation of $\Delta_{y} \cap \mathrm{III}^{8}(f)$.

Lemma 6.3 The incidence coefficient $\left[\mathrm{III}^{8}: \mathfrak{F}\right]$ vanishes for every $C^{0}$ equivalence class modulo regular fibers $\mathfrak{F}$ of codimension 4 that is chiral.

Proof It is not difficult to see that for $y \in \mathfrak{F}(f), \Delta_{y} \cap \mathrm{III}^{8}(f) \neq \varnothing$ if and only if $\mathfrak{F}=\mathrm{IV}^{0,8}, \mathrm{IV}^{1,8}$ or $\mathrm{IV}^{18}$. Furthermore, for each of these three cases, the number of arcs of $\Delta_{y} \cap \mathrm{III}^{8}(f)$ is equal to 2 and exactly one of them is coming into $y$. Thus the result follows.

Remark 6.4 The above lemma shows that the closure of $\operatorname{III}^{8}(f)$ is a regular oriented 1-dimensional submanifold of $N$ near the points over which lies a chiral singular fiber of codimension 4 . However, the closure of III $^{8}(f)$, as a whole, is not even a topological manifold in general. For example, suppose that $f$ admits a $\mathrm{IV}^{22}$ type fiber. Then the closure of $\operatorname{III}^{8}(f)$ forms a graph (that is, a 1-dimensional complex) and each point of $\mathrm{IV}^{22}(f)$ is a vertex of degree 8 , that is, it has 8 incident edges. Furthermore, four of them are incoming edges and the other four are outgoing edges. 
Let us recall the following definition (for details, refer to Conner-Floyd [3].)

Definition 6.5 Let $N$ be a manifold and $f_{i}: M_{i} \rightarrow N$ a continuous map of a closed oriented $n$-dimensional manifold $M_{i}$ into $N, i=0,1$. We say that $f_{0}$ and $f_{1}$ are oriented bordant if there exist a compact oriented $(n+1)$-dimensional manifold $W$ and a continuous map $F: W \rightarrow N \times[0,1]$ with the following properties:

(1) $\partial W$ is identified with the disjoint union of $-M_{0}$ and $M_{1}$, where $-M_{0}$ denotes the manifold $M_{0}$ with the reversed orientation, and

(2) $\left.F\right|_{M_{i}}: M_{i} \rightarrow N \times\{i\}$ is identified with $f_{i}, i=0,1$.

We call the map $F: W \rightarrow N \times[0,1]$ an oriented bordism between $f_{0}$ and $f_{1}$.

Note that if $M_{0}=M_{1}$, and $f_{0}$ and $f_{1}$ are homotopic, then they are oriented bordant. Furthermore, if the target manifold $N$ is contractible, then $f_{0}$ and $f_{1}$ are oriented bordant if and only if their source manifolds $M_{0}$ and $M_{1}$ are oriented cobordant as oriented manifolds.

For a given manifold $N$ and a nonnegative integer $n$, the set of all oriented bordism classes of maps of closed oriented $n$-dimensional manifolds into $N$ forms an additive group under the disjoint union. We call it the $n$-dimensional oriented bordism group of $N$.

Note that in the usual definition, an oriented bordism is a map into $N$ and not into $N \times[0,1]$. However, it is easy to see that the above definition is equivalent to the usual one.

As a consequence of Lemma 6.3, we get the following.

Lemma 6.6 Let $N$ be a 3-manifold and $f_{i}: M_{i} \rightarrow N$ a $C^{\infty}$ stable map of a closed oriented 4-manifold $M_{i}$ into $N, i=0,1$. If $f_{0}$ and $f_{1}$ are oriented bordant, then we have

$$
\left\|\mathrm{III}^{8}\left(f_{0}\right)\right\|=\left\|\mathrm{III}^{8}\left(f_{1}\right)\right\| .
$$

Proof Let $F: W \rightarrow N \times[0,1]$ be an oriented bordism between $f_{0}$ and $f_{1}$. Take sufficiently small collar neighborhoods $C_{0}=M_{0} \times[0,1)$ and $C_{1}=M_{1} \times(0,1]$ of $M_{0}$ and $M_{1}$ in $W$ respectively. We may assume that

$$
\begin{aligned}
\left.F\right|_{M_{0} \times[0, \varepsilon)} & =f_{0} \times \mathrm{id}_{[0, \varepsilon)}, \quad \text { and } \\
\left.F\right|_{M_{1} \times(1-\varepsilon, 1]} & =f_{1} \times \mathrm{id}_{(1-\varepsilon, 1]}
\end{aligned}
$$

for a sufficiently small $\varepsilon>0$. Furthermore, we may assume that $F$ is a smooth map with $F^{-1}(N \times(0,1))=\operatorname{Int} W$. Then by a standard argument, we can approximate $F$ by a 
generic map $F^{\prime}$ such that $\left.F^{\prime}\right|_{C_{0} \cup C_{1}}=\left.F\right|_{C_{0} \cup C_{1}}$ and that $\left.F^{\prime}\right|_{\text {Int } W}$ : Int $W \rightarrow N \times(0,1)$ is a proper $C^{\infty}$ stable map. In the following, let us denote $F^{\prime}$ again by $F$.

By Lemma 6.3, we see that the closure of $\operatorname{III}^{8}(F)$ is a finite graph each of whose edge is oriented. Furthermore, for each vertex lying in $N \times(0,1)$, the number of incoming edges is equal to that of outgoing edges. Furthermore, its vertices lying in $N \times\{0,1\}$ have degree one and they coincide exactly with the union of

$$
\begin{aligned}
& \mathrm{III}^{8}(F) \cap(N \times\{0\})=\operatorname{III}^{8}\left(f_{0}\right) \quad \text { and } \\
& \mathrm{III}^{8}(F) \cap(N \times\{1\})=\operatorname{III}^{8}\left(f_{1}\right) .
\end{aligned}
$$

Therefore, by virtue of Remark 5.2 we have

$$
-\left\|\mathrm{III}^{8}\left(f_{0}\right)\right\|+\left\|\mathrm{III}^{8}\left(f_{1}\right)\right\|=0,
$$

since $\partial W=\left(-M_{0}\right) \cup M_{1}$. Hence the result follows.

By combining Lemma 6.6 with a work of Conner-Floyd [3], we get the following.

Proposition 6.7 Let $N$ be a 3-manifold and $f_{i}: M_{i} \rightarrow N$ a $C^{\infty}$ stable map of a closed oriented 4-manifold $M_{i}$ into $N, i=0,1$. If $M_{0}$ and $M_{1}$ are oriented cobordant as oriented 4-manifolds, then we have

$$
\left\|\mathrm{III}^{8}\left(f_{0}\right)\right\|=\left\|\mathrm{III}^{8}\left(f_{1}\right)\right\| .
$$

Proof Recall that the oriented cobordism groups $\Omega_{n}$ of $n$-dimensional manifolds for $0 \leq n \leq 4$ satisfy the following:

$$
\Omega_{n} \cong \begin{cases}0, & n=1,2,3, \\ \mathbb{Z}, & n=0,4 .\end{cases}
$$

Furthermore, the 4-dimensional oriented bordism group of $N$ is isomorphic to

$$
\sum_{p+q=4} H_{p}\left(N ; \Omega_{q}\right)
$$

modulo (odd) torsion [3, Section 15]. Therefore, if the 4-dimensional manifolds $M_{0}$ and $M_{1}$ are oriented cobordant, then $m f_{0}$ and $m f_{1}$ are oriented bordant for some odd integer $m$, where $m f_{i}$ denotes the map of the disjoint union of $m$ copies of $M_{i}$ into $N$ such that on each copy it is given by $f_{i}, i=0,1$.

Thus by Lemma 6.6, we have

$$
m\left\|\operatorname{III}^{8}\left(f_{0}\right)\right\|=m\left\|\operatorname{III}^{8}\left(f_{1}\right)\right\|,
$$

which implies the desired equality. This completes the proof. 


\section{An explicit example}

In this section, we study an explicit example of a $C^{\infty}$ stable map of a closed oriented 4-manifold with nonzero signature into $\mathbb{R}^{3}$. Combining this with Proposition 6.7, we will prove our main theorem of this paper.

In [24, Chapter 6], the first named author constructed an explicit example of a $C^{\infty}$ stable map $f: M \rightarrow \mathbb{R}^{3}$ of a closed 4-manifold $M$ with exactly one III $^{8}$ type fiber such that $f$ has only fold points as its singularities. Recall that $M$ is diffeomorphic to $\mathbb{C} P^{2} \sharp 2 \overline{\mathbb{C} P^{2}}$ if we ignore the orientation. We would like to orient $M$ and determine the sign of this $\mathrm{III}^{8}$ type fiber.

In fact, by Proposition 6.7, we already know that there exists a constant $c$ such that the algebraic number of III $^{8}$ type fibers is $c$ times the signature of the source oriented 4-manifold (for details, see the argument in the proof of Theorem 5.5 below). In the above-mentioned example, the algebraic number of III $^{8}$ type fibers is equal to \pm 1 , and the signature of the source 4-manifold is equal to \pm 1 . Therefore, this constant $c$ should be equal to \pm 1 . Thus, for the proof of our main theorem, it suffices to determine the sign of the constant $c$.

This procedure might seem easy, but in fact it is not. As a matter of fact, the construction of the above example in [24] was already very complicated, although the example itself seems to be a natural one. Therefore, in this section, we carefully study the example and determine the sign of the constant $c$. We will describe the argument in details, since the technique in this section can be very useful in determining the self-intersection number of the surface of singular point set in general situations. At the end of this section, we give a new proof of the self-intersection number formula based on our study.

In the construction given in [24], the orientation of the source 4-manifold $M$ was not given explicitly. Here, we first orient the source $4-$ manifold so that the III $^{8}$ type fiber gets the positive sign, and then determine the signature of the source 4-manifold with respect to the chosen orientation.

Lemma 7.1 If we orient the source 4-manifold $M$ of the above example so that the III $^{8}$ type fiber is positive, then the signature of $M$ is equal to +1 .

Proof In general, let $f: M \rightarrow \mathbb{R}^{3}$ be a $C^{\infty}$ stable map of a closed oriented 4manifold into the 3-dimensional Euclidean space which has only fold points as its singularities. In view of a result in the first named author's paper [23] (see also Ohmoto-Saeki-Sakuma [19] or Sadykov [21]), we have

$$
3 \sigma(M)=S_{0}(f) \cdot S_{0}(f),
$$


where $S_{0}(f)$ denotes the surface of definite fold singularities of $f$, and $S_{0}(f) \cdot S_{0}(f)$ denotes its self-intersection number (or equivalently, its normal Euler number) in $M$. Therefore, in order to determine the signature of the source 4-manifold of the explicit example mentioned above, we have only to compute the self-intersection number $S_{0}(f) \cdot S_{0}(f)$.

Let us first consider the $\mathrm{III}^{8}$ type fiber and denote the three singular points in it by $q_{1}$, $q_{2}$ and $q_{3}$ as in Figure 9. Furthermore, we orient the regular part of the III $^{8}$ type fiber so that it corresponds to the cyclic order $\left\langle q_{1}, q_{2}, q_{3}\right\rangle$ of the three singular points.

The image $f(S(f))$ of the singular point set of $f$ around the point $y$ corresponding to the III $^{8}$ type fiber consists of three sheets $f\left(D_{1}\right), f\left(D_{2}\right)$ and $f\left(D_{3}\right)$, where $D_{i}$ is a small disk neighborhood of $q_{i}$ in $S(f), i=1,2,3$. We may assume that the three sheets $f\left(D_{1}\right), f\left(D_{2}\right)$ and $f\left(D_{3}\right)$ are situated as in Figure 10 and that the "front octant" $\omega$ in the figure is a 1 -octant; that is, the fiber over a point in the octant is connected.

Let $w_{i}$ be a normal vector to the $i$-th sheet of $f(S(f))$ in $N$ pointing toward $\omega$ at a point incident to that octant, $i=1,2,3$. We orient $\mathbb{R}^{3}$ so that the ordered 3-tuple of vectors $\left\langle w_{1}, w_{2}, w_{3}\right\rangle$ is consistent with the orientation, that is, $\mathbb{R}^{3}$ is endowed with the "right-handed orientation" in the usual sense.

Now we orient the source 4-manifold of $f$ so that the regular part of the III $^{8}$ type fiber gets the orientation as indicated in Figure 9 in the sense of Section 5 by the "fiber first" convention. Then the sign of the III $^{8}$ type fiber is equal to +1 .

Recall that $S_{0}(f)$ consists of three $2-$ spheres $\widetilde{S}_{0}, \widetilde{S}_{1}$ and $\widetilde{S}_{2}$, and that the surface $S_{1}(f)$ of indefinite fold points is a real projective plane whose image $P=f\left(S_{1}(f)\right)$ is Boy's surface in $\mathbb{R}^{3}$ (see Figure 12). Furthermore, the embedded 2-sphere $S_{0}=f\left(\widetilde{S}_{0}\right)$ surrounds Boy's surface, and the disjoint 2 -spheres $S_{1}=f\left(\widetilde{S}_{1}\right)$ and $S_{2}=f\left(\widetilde{S}_{2}\right)$ are contained in the bounded region of $\mathbb{R}^{3} \backslash P$ so that $S_{1}$ surrounds $S_{2}$ (for details, see [24, Chapter 6]).

We have obviously a continuous map $h_{0}: S_{0} \times[0,1] \rightarrow \mathbb{R}^{3}$ with the following properties:

(1) $\left.h_{0}\right|_{S_{0} \times\{0\}}=\operatorname{id}_{S_{0}}: S_{0} \times\{0\} \rightarrow S_{0}$.

(2) $\left.h_{0}\right|_{S_{0} \times(0,1)}$ is a diffeomorphism onto the region bounded by $S_{0}$ and $P$.

(3) $h_{0}\left(S_{0} \times\{1\}\right)=P$.

(4) $\left.h_{0}\right|_{S_{0} \times\{1\}}$ is a homeomorphism outside of a 1-dimensional subcomplex $C_{0}$ of $S_{0}$ as depicted in Figure 13. The image $h_{0}\left(\left(S_{0} \backslash C_{0}\right) \times\{1\}\right)$ coincides with the complement to the multiple point set of Boy's surface $P$ in $P$. 

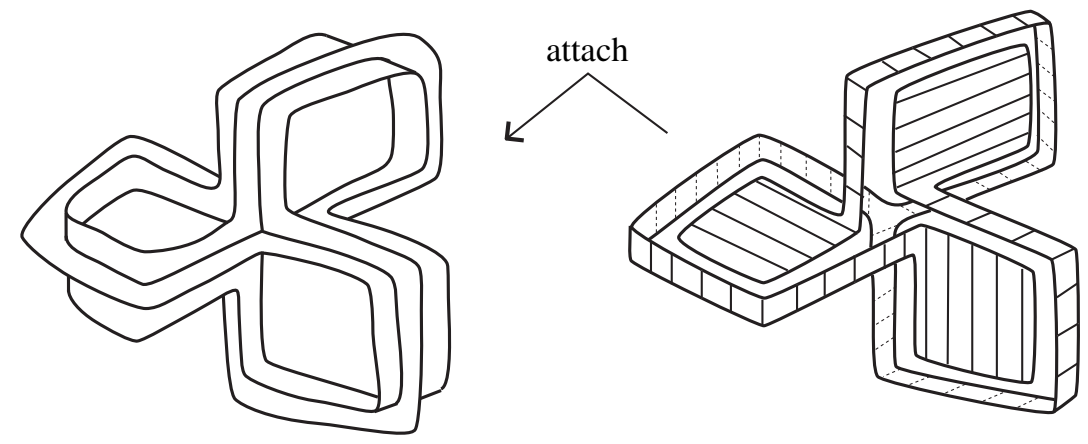

Figure 12: Boy's surface $P$

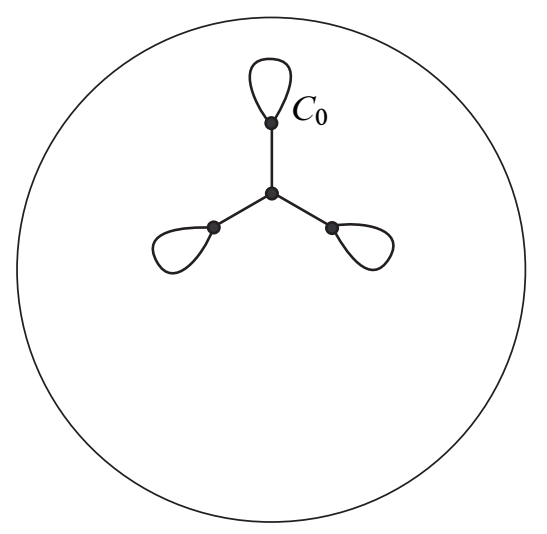

Figure 13: 1-dimensional subcomplex $C_{0}$ on the 2 -sphere $S_{0}$

Set $B=h_{0}\left(S_{0} \times[0,1 / 2]\right)$. Then $N\left(\widetilde{S}_{0}\right)=f^{-1}(B)$ can be identified with a normal disk bundle $v_{0}$ to $\widetilde{S}_{0}$ in $M$. In order to calculate the self-intersection number $\widetilde{S}_{0} \cdot \widetilde{S}_{0}$ in $M$, let us consider the disk bundle $\widetilde{v}_{0}$ over $\widetilde{S}_{0}$ which is obtained from $v_{0}$ by identifying the antipodal points on each disk fiber. In other words, the $S^{1}$-bundle $\partial \widetilde{v}_{0}$ associated with the disk bundle $\widetilde{v}_{0}$ corresponds to the $\mathbb{R} P^{1}$-bundle associated with the real 2-plane bundle afforded by $v_{0}$. Note that the self-intersection number of the zero section of $v_{0}$ is equal to one half of the self-intersection number of that of $\widetilde{v}_{0}$.

Let us construct a section of the $S^{1}$-bundle $\partial \widetilde{v}_{0}$ associated with $\widetilde{v}_{0}$ over a certain subset $\tilde{X}_{0}$ of $\tilde{S}_{0}$. More precisely, for each point $\tilde{x}$ of $\tilde{X}_{0}$, we will choose a pair of antipodal points on the circle fiber of $\partial v_{0}$ over $\tilde{x}$ continuously with respect to $\tilde{x}$ so that the projection restricted to the set of all these points is a double covering map onto $\tilde{X}_{0}$, where $\partial v_{0}$ is the $S^{1}$-bundle associated with the disk bundle $v_{0}$. 
Let $N\left(C_{0}\right)$ be a regular neighborhood of $C_{0}$ in $S_{0}$. Set $\widetilde{C}_{0}=f^{-1}\left(C_{0}\right)$ and $N\left(\widetilde{C}_{0}\right)=$ $f^{-1}\left(N\left(C_{0}\right)\right)$. Note that $\widetilde{C}_{0}$ is a 1 -dimensional subcomplex of $\widetilde{S}_{0}$ and that $N\left(\widetilde{C}_{0}\right)$ is a regular neighborhood of $\widetilde{C}_{0}$ in $\widetilde{S}_{0}$. We will first construct a section of the $S^{1}$-bundle $\partial \widetilde{v}_{0}$ over $\widetilde{S}_{0} \backslash \operatorname{Int} N\left(\widetilde{C}_{0}\right)\left(\subset \widetilde{X}_{0}\right)$ as follows.

Take a point $\tilde{x} \in \widetilde{S}_{0} \backslash \operatorname{Int} N\left(\widetilde{C}_{0}\right)$ and set $x=f(\tilde{x}) \in S_{0}$. Then $h_{0}(x, 1) \in P$ is the image of a unique indefinite fold point. Therefore, $f^{-1}\left(h_{0}(x, 1 / 2)\right)$ can be considered as a nearby fiber of the fiber over $h_{0}(x, 1)$, which is of type $\mathrm{I}^{1}$, and hence we can take a pair of two antipodal points on $f^{-1}\left(h_{0}(x, 1 / 2)\right)$ canonically as in Figure 14 . We can thus construct a continuous section of $\partial \widetilde{v}_{0}$ over each component of $\widetilde{S}_{0} \backslash \operatorname{Int} N\left(\widetilde{C}_{0}\right)$.

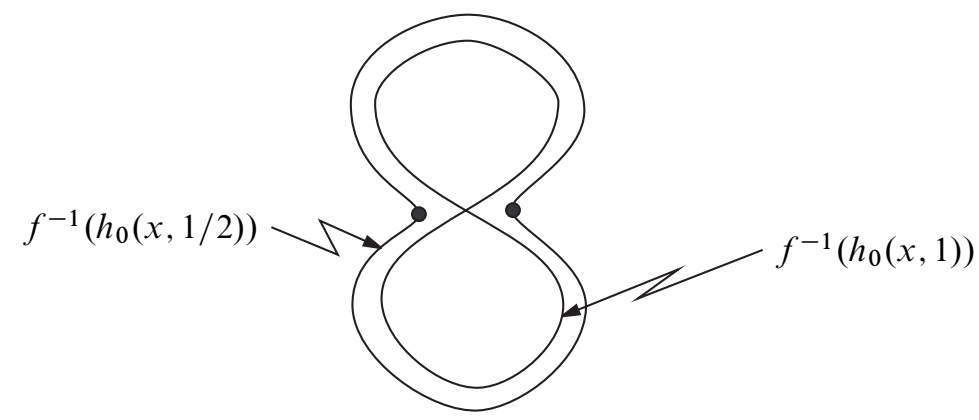

Figure 14: Two points on a regular fiber near a singular fiber of type $\mathrm{I}^{1}$

Let us consider the twelve bands embedded in $N\left(\widetilde{C}_{0}\right)$ as in Figure 15, where each band is homeomorphic to $[-1,1] \times[-1,1]$. Each band is also considered to be a $1-$ handle attached to $\widetilde{S}_{0} \backslash \operatorname{Int} N\left(\widetilde{C}_{0}\right)$ along $\{-1,1\} \times[-1,1]$. We orient the core of each band as in the figure, where a core is an arc properly embedded in a band corresponding to $[-1,1] \times\{0\}$. The subset $\tilde{X}_{0}$ is the union of $\widetilde{S}_{0} \backslash \operatorname{Int} N(\widetilde{C})$ and the twelve bands. Let us extend the section of $\partial \widetilde{v}_{0}$ over $\widetilde{S}_{0} \backslash \operatorname{Int} N\left(\widetilde{C}_{0}\right)$ constructed above through the twelve bands as follows.

Take a band and let $\tilde{\alpha}$ be its core. Set $\alpha=f(\widetilde{\alpha})$. Since $\alpha^{\prime}=h_{0}(\alpha \times\{1 / 2\})$ is close to the transverse double point of $f(S(f))$ as in Figure 1 (2), the regular fibers over the two points $\partial \alpha^{\prime}$ are close to a $\mathrm{II}^{3}$-fiber. Furthermore, the pairs of antipodal points on the circle fibers over the two points $\partial \widetilde{\alpha}$ associated with the above-constructed section of $\partial \widetilde{v}_{0}$ over $\widetilde{S}_{0} \backslash \operatorname{Int} N\left(\widetilde{C}_{0}\right)$ are situated as in Figure 16. Let us extend the section of $\partial \widetilde{v}_{0}$ over $\widetilde{S}_{0} \backslash \operatorname{Int} N\left(\widetilde{C}_{0}\right)$ through $\widetilde{\alpha}$ so that when one goes along $\widetilde{\alpha}$ in the direction indicated as in Figure 15, the pair of antipodal points on the circle fibers of $\partial v_{0}$ gets the rotation through the angle $\pi / 2$ in the positive direction of regular fibers. Then we can naturally extend the section to the band. We apply this construction to all the twelve bands to get a section of $\partial \widetilde{v}_{0}$ over $\tilde{X}_{0}$. 


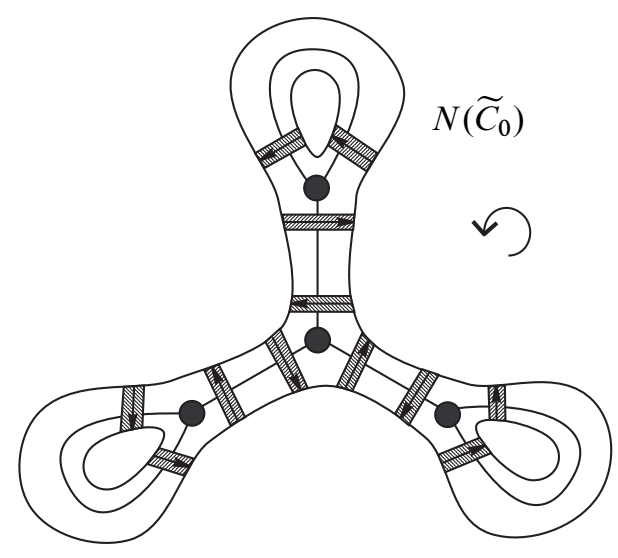

Figure 15: Twelve bands in $N\left(\widetilde{C}_{0}\right)$
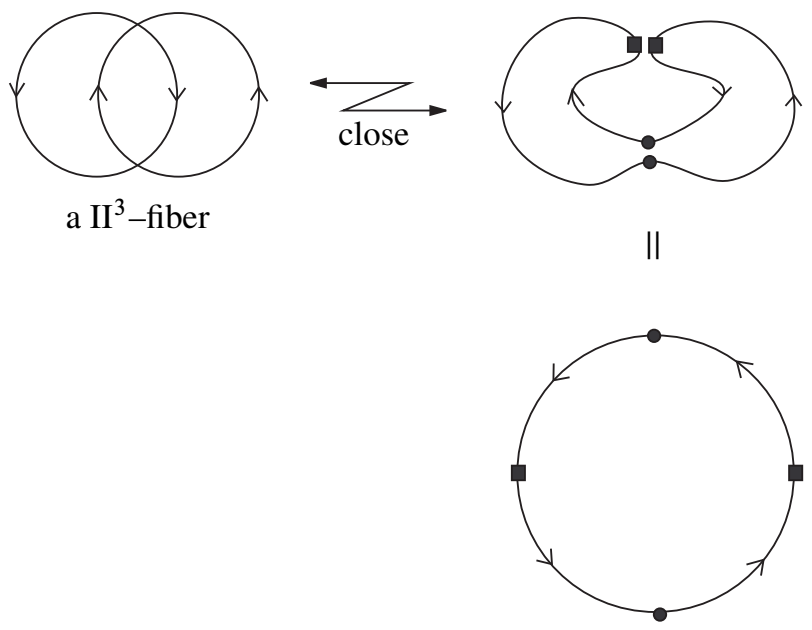

Figure 16: Two pairs of antipodal points

The complement of Int $\widetilde{X}_{0}$ in $\widetilde{S}_{0}$ consists of ten 2-disks: six rectangular disks corresponding to the edges of $\widetilde{C}_{0}$ and four hexagonal disks corresponding to the vertices of $\widetilde{C}_{0}$. Note that the bundle $\widetilde{v}_{0}$ over each 2 -disk $\Delta$ is trivial, and using a trivialization, we can define the degree of the above-constructed section over $\partial \Delta$. The self-intersection number of the zero section of $\widetilde{v}_{0}$ is then equal to the sum of these "degrees" over the boundaries of the ten 2 -disks. Note that here $\widetilde{S}_{0}$ should be oriented so that its orientation together with the orientation of the 2-disk fiber gives the orientation of 
the total space. Since we have oriented the source 4-manifold by the "fiber first" convention, we may assume that

(i) the induced orientation on a 2-disk fiber of the normal disk bundle $v_{0}$ to $\widetilde{S}_{0}$ in $M$ is given by the orientation of the boundary regular fiber plus the "inward normal", and

(ii) the induced orientation $\mathcal{O}_{S_{0}}$ on $S_{0}$ satisfies that the "outward normal" plus $\mathcal{O}_{S_{0}}$ is consistent with the right-handed orientation of $\mathbb{R}^{3}$.

Hence $\mathcal{O}_{S_{0}}$ is the "left-handed" orientation when viewed from outside (see Figure 15). Here we adopt the convention that the figure of $N\left(\widetilde{C}_{0}\right)$ (Figure 15) is consistent with that of $N\left(C_{0}\right)$ viewed from outside.

It is easy to see that for each of the six rectangular 2-disks of $\tilde{S}_{0} \backslash$ Int $\tilde{X}_{0}$, the degree of the section of $\partial \widetilde{v}_{0}$ over its boundary is equal to -1 , since when we go around its boundary in its positive direction, the pair of antipodal points on the circle fibers of $\partial v_{0}$ rotates through the angle $-\pi$.

Let us now consider the contribution of each of the other four regions of $\widetilde{S}_{0} \backslash$ Int $\tilde{X}_{0}$ that are hexagonal. The image $H$ by $h_{0}(*, 1 / 2)$ of its $f$-image is close to the triple point $y$ of $f(S(f))$ and it lies in a 1-octant (see Figure 10). Recall that $H$ is given the "left-handed" orientation when viewed from outside. Therefore, when we go along the boundary of the hexagonal disk $H$ in the positive direction from a point near the sheet $f\left(D_{1}\right)$, then the second sheet that we pass by is the sheet $f\left(D_{2}\right)$. In this process, the pair of antipodal points on a circle fiber of $\partial v$ corresponding to the sheet $f\left(D_{1}\right)$ makes a rotation through the angle $\pi / 3$ as in Figure 17, since the sign of the III $^{8}$ type fiber is positive. Therefore, the degree of the section of $\partial \widetilde{v}_{0}$ over the boundary of the hexagonal disk is equal to +1 , since when we go around its boundary in its positive direction, the pair of antipodal points on the circle fibers of $\partial v_{0}$ rotates through the angle $\pi$ (see Figure 17). We also note that this argument can be equally applied to all the four hexagonal regions (that is, the central hexagonal region is not an exception), since the argument is local in nature in the target.

Thus the sum of the degrees is equal to

$$
(-1) \cdot 6+(+1) \cdot 4=-2 .
$$

Therefore, the self-intersection number of the zero section of $\widetilde{v}_{0}$ is equal to -2 and that of $v_{0}$ is equal to $-2 / 2=-1$.

The self-intersection numbers $\widetilde{S}_{1} \cdot \widetilde{S}_{1}$ and $\widetilde{S}_{2} \cdot \widetilde{S}_{2}$ can be computed by a similar method as follows. Let $v_{i}$ be the normal disk bundle to $\widetilde{S}_{i}$ in $M, i=1,2$. We can construct 

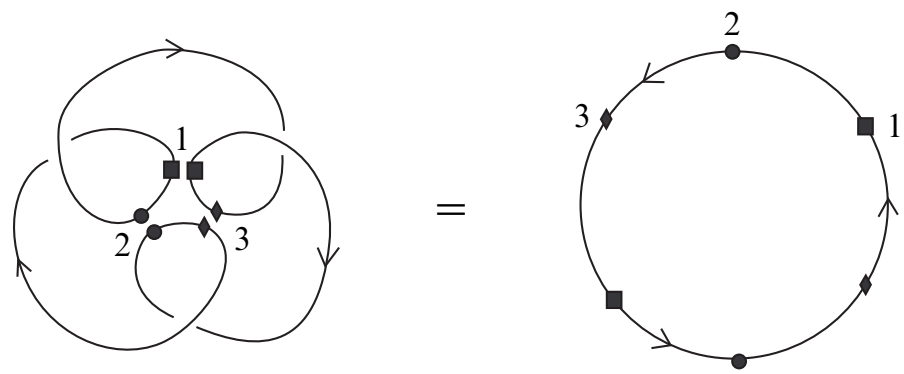

Figure 17: Three pairs of antipodal points

the continuous map $h_{i}, i=1,2$, for $f\left(\widetilde{S}_{i}\right)=S_{i}$ satisfying the properties similar to those for $h_{0}$. Then we take the 1 -dimensional complexes $C_{i}$ and $\tilde{C}_{i}$, their regular neighborhoods $N\left(C_{i}\right)$ and $N\left(\widetilde{C}_{i}\right)$ respectively, and the twelve bands in $\widetilde{S}_{i}, i=1,2$, as above, and define $\widetilde{X}_{i}$ to be the union of $\widetilde{S}_{i} \backslash \operatorname{Int} N\left(\widetilde{C}_{i}\right)$ and the twelve bands. Then we can construct a section of $\partial v_{i}, i=1,2$, over $\tilde{X}_{i}$ by using an argument similar to that for $\partial \widetilde{v}_{0}$. (Note that in the present case, we use $v_{i}$ itself, rather than its associated $\mathbb{R} P^{1}$-bundle.) More precisely, let us consider the regular fiber over the point $h_{i}(x, 1 / 2)$ for a point $\tilde{x} \in \widetilde{S}_{i} \backslash \operatorname{Int} N\left(\widetilde{C}_{i}\right)$ with $x=f(\tilde{x})$. It consists of two circles $\ell_{1}$ and $\ell_{2}$ and we can take a pair of points which lie on distinct components as in Figure 18.

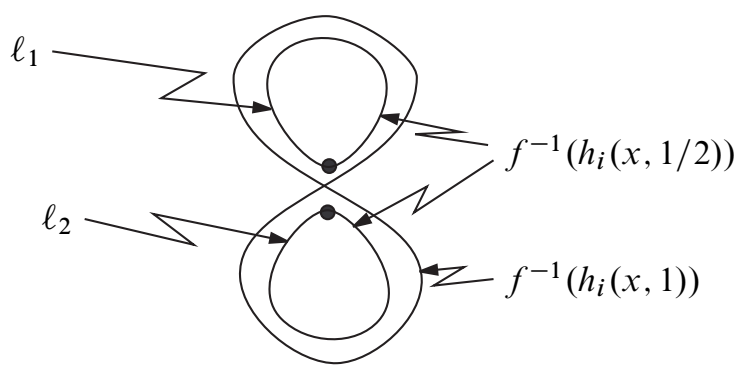

Figure 18: Two points on a regular fiber which are on distinct connected components

We can extend this section through the twelve bands as in the case of $\widetilde{S}_{0}$. Let us compute the sum of the degrees of the section over the components of $\partial \widetilde{X}_{i}$. First note that the orientation on $S_{i}$ is the "left-handed" orientation when viewed from inside. Then the contribution of each rectangular region is equal to +1 , since when we go along the core of a band, the chosen point on a connected component of a regular fiber gets the rotation through the angle $\pi$ (see Figure 19).

As to the other four hexagonal regions, they correspond to the triple point of $f(S(f))$. When we go around the boundary, the chosen point gets the rotation through the angle 

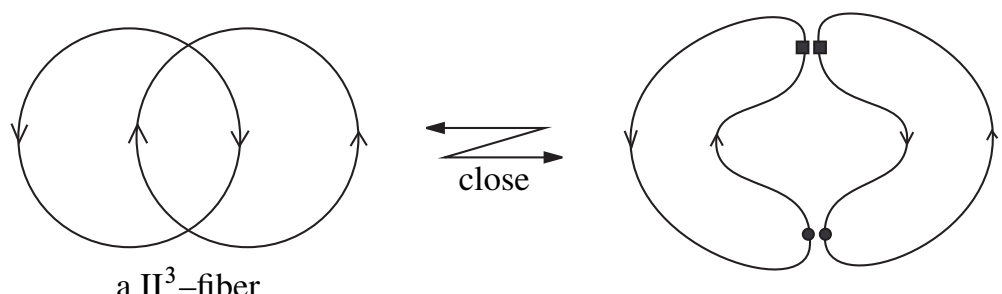

Figure 19: Two pairs of points

$-2 \pi$ (see Figure 20). Hence its contribution to the self-intersection number is equal to -1 .

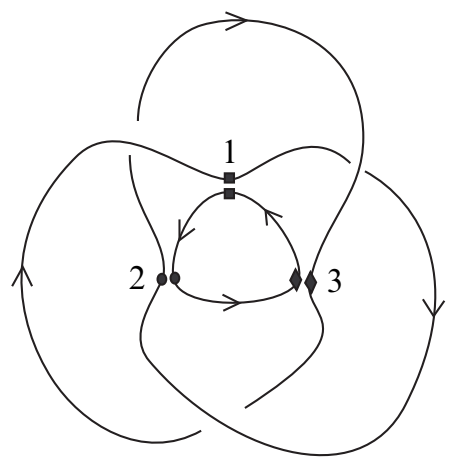

Figure 20: Three pairs of points

Therefore, the self-intersection number $\widetilde{S}_{i} \cdot \widetilde{S}_{i}$ is equal to

$$
(+1) \cdot 6+(-1) \cdot 4=2
$$

for $i=1,2$.

Thus the self-intersection number of $S_{0}(f)$ in $M$ is equal to

$$
S_{0}(f) \cdot S_{0}(f)=\widetilde{S}_{0} \cdot \widetilde{S}_{0}+\widetilde{S}_{1} \cdot \widetilde{S}_{1}+\widetilde{S}_{2} \cdot \widetilde{S}_{2}=-1+2+2=3 .
$$

Therefore, the signature of the source 4 -manifold is equal to +1 according to the formula (7-1). In other words, the source 4-manifold $M$ is oriented diffeomorphic to $2 \mathbb{C} P^{2} \sharp \overline{\mathbb{C} P^{2}}$. This completes the proof of Lemma 7.1.

Let us now proceed to the proof of our main theorem of this paper. 
Proof of Theorem 5.5 Let us fix a 3-manifold $N$. For a closed oriented 4-manifold $M$ and a $C^{\infty}$ stable map $f: M \rightarrow N$ into $N$, let us consider the integer

$$
\psi(M, f)=\left\|\mathrm{III}^{8}(f)\right\| .
$$

By virtue of Proposition 6.7, $\psi$ depends only on the oriented cobordism class of $M$. Therefore, $\psi$ induces a map

$$
\bar{\psi}: \Omega_{4} \rightarrow \mathbb{Z}
$$

of the 4-dimensional oriented cobordism group to the additive group of integers. This is clearly a homomorphism.

Recall that $\Omega_{4}$ is an infinite cyclic group generated by the class of oriented 4-manifolds with signature +1 . In other words, the signature induces an isomorphism

$$
\sigma: \Omega_{4} \rightarrow \mathbb{Z}
$$

Let us consider the explicit example $f: 2 \mathbb{C} P^{2} \sharp \overline{\mathbb{C} P^{2}} \rightarrow \mathbb{R}^{3}$ given in Lemma 7.1. By composing it with an embedding $\mathbb{R}^{3} \hookrightarrow N$, we get a $C^{\infty}$ stable map of an oriented 4-manifold of signature +1 into $N$. This stable map has exactly one III $^{8}$ type fiber, whose sign is equal to +1 . Therefore, the homomorphism

$$
\bar{\psi} \circ \sigma^{-1}: \mathbb{Z} \rightarrow \mathbb{Z}
$$

sends +1 to +1 and hence is the identity. Thus we have $\sigma=\bar{\psi}$. This completes the proof.

We have a direct consequence of our main theorem as follows.

Corollary 7.2 Let $M$ be a closed oriented 4-manifold and $N$ a 3-manifold. Then every $C^{\infty}$ stable map of $M$ into $N$ has at least $|\sigma(M)|$ singular fibers of type III $^{8}$.

For example, if $M$ is the underlying real 4-dimensional manifold of the complex K3 surface, then every $C^{\infty}$ stable map of $M$ into a 3-manifold has at least 16 fibers of type III $^{8}$, although no explicit example of such a map is known. Construction of an explicit example can be an interesting problem.

Our study of the explicit example gives a new proof to the following formula, which has been proved by Ohmoto-Saeki-Sakuma [19] and Sadykov [21].

Corollary 7.3 Let $f: M \rightarrow N$ be a $C^{\infty}$ stable map of a closed oriented 4-manifold $M$ into an orientable 3-manifold $N$. Then we have

$$
S(f) \cdot S(f)=3 \sigma(M)
$$


where $S(f) \cdot S(f)$ is the self-intersection number of the surface of singular point set of $f$ in $M$.

Note that the formula (7-1) follows from Corollary 7.3. We give a proof to the above corollary without using the formula (7-1).

Proof of Corollary 7.3 It is not difficult to show that the self-intersection number $S(f) \cdot S(f)$ is an oriented cobordism invariant of the source 4-manifold $M$ (for this, use the argument as in [21, Proof of Lemma 3.2] together with a result of Conner-Floyd [3] as in the proof of Proposition 6.7). Therefore, there exists a constant $c$ such that

$$
S(f) \cdot S(f)=3 \operatorname{co}(M)
$$

holds for any $C^{\infty}$ stable map $f$ of a closed oriented 4-manifold $M$ into an orientable 3-manifold $N$.

For the explicit example $f$ studied above, we have ${ }^{2} S(f) \cdot S(f)= \pm 3$ and $3 \sigma(M)=$ \pm 3 . Therefore, the constant $c$ must be equal to \pm 1 .

On the other hand, by a result of Sakuma [25] we have

$$
S(f) \cdot S(f) \equiv 3 \sigma(M) \quad(\bmod 4) .
$$

Therefore, the constant $c$ must be equal to +1 . This completes the proof.

Recall that Sadykov [21] proved Corollary 7.3 by a characteristic class argument together with Sakuma's result [25].

Now some remarks concerning our result are in order.

Remark 7.4 For a closed oriented 4-manifold $M$, let $n_{+}$and $n_{-}$be arbitrary integers such that $\sigma(M)=n_{+}-n_{-}$. Then does there exist a $C^{\infty}$ stable map $f: M \rightarrow N$ that has exactly $n_{+}$positive singular fibers of III $^{8}$ type and $n_{-}$negative ones? The authors do not know the answer to the question.

Remark 7.5 In [22] the first named author proved that if $f: M \rightarrow N$ is a $C^{\infty}$ stable map of a closed oriented 4-manifold into a 3-manifold with only definite fold singularities, then $\sigma(M)=0$. This follows from our main theorem as well.

Remark 7.6 The technique of this section to determine the self-intersection number of the surface of definite fold points may be generalized in a more general setting. This might give a new direct proof of our main theorem.

\footnotetext{
${ }^{2}$ We know that $S(f) \cdot S(f)=3 \sigma(M)=3$ : however, in order to show this, we used the formula (7-1). Here we are proving the corollary without using (7-1).
} 
Remark 7.7 It is known that there exist oriented surface bundles over oriented surfaces with non-zero signatures (for example, see Meyer [17]). This means that we cannot expect a similar signature formula for $C^{\infty}$ stable maps of closed oriented 4-manifolds into surfaces.

Note that if the fiber genus $g \leq 2$, then there is a signature formula for Lefschetz fibrations in terms of singular fibers (see the work of Matsumoto [15; 16]). We also have a similar formula for hyperelliptic Lefschetz fibrations of any genus (see Endo [6]). It may be possible to prove these formulas by using our main theorem as follows. Let $M$ be a Lefschetz fibration over a surface $B$, and consider a line bundle $N$ over $B$. Note that $N$ is a 3 -manifold. Then we can consider a generic map $f: M \rightarrow N$ which makes the diagram

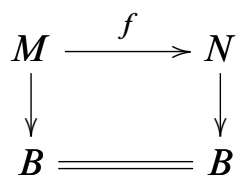

commutative, where the vertical arrows are relevant fibration maps. In other words, we consider a generic family of fiberwise functions. By applying our Theorem 5.5 to $f$, we might be able to get a signature formula for certain Lefschetz fibrations.

\section{Universal complex of chiral singular fibers}

In [24], the universal complex of singular fibers was introduced as a refinement of Vassiliev's universal complex of multi-singularities (see Vassiliev [26], Kazaryan [9] or Ohmoto [18]), and it was shown that its cohomology classes give invariants of cobordisms of singular maps in the sense of Rimányi and Szúcs [20]. In this section, we study the universal complex (with integer coefficients) of singular fibers corresponding to chiral singular fibers and give an interpretation of our main theorem in terms of the theory of universal complex of singular fibers.

We can define the universal complex of chiral singular fibers for proper $C^{\infty}$ stable maps of oriented 5-manifolds into 4-manifolds by exactly the same procedure as in [24] as follows.

For $\kappa$ with $3 \leq \kappa \leq 4$, let $C^{\kappa}$ be the free $\mathbb{Z}$-module generated by the $C^{0}$ equivalence classes modulo regular fibers of chiral singular fibers of codimension $\kappa$. Note that rank $C^{3}=3$ and $\operatorname{rank} C^{4}=14$ according to Proposition 6.1. Since there exist no chiral singular fibers of codimension $\kappa \neq 3$, 4 , we put $C^{\kappa}=0$ for $\kappa \neq 3,4$. Note that for $\kappa=4$, we take the $C^{0}$ equivalence classes modulo regular fibers of chiral singular 
fibers with positive signs as generators, and we consider those with negative signs to be -1 times the corresponding class with positive sign.

The coboundary homomorphism $\delta_{3}: C^{3} \rightarrow C^{4}$ is defined by

$$
\delta_{3}(\mathfrak{G})=\sum_{\kappa(\mathfrak{F})=4}[\mathfrak{G}: \mathfrak{F}] \mathfrak{F}
$$

for every generator $\mathfrak{G}$ of $C^{3}$, where $[\mathfrak{G}: \mathfrak{F}] \in \mathbb{Z}$ is the incidence coefficient which can be defined by exactly the same method as for $\left[\mathrm{III}^{8}: \mathfrak{F}\right]$ (see Section 6 ). Note that all the other coboundary homomorphisms $\delta_{\kappa}, \kappa \neq 3$, are necessarily trivial.

We call the resulting cochain complex $\left(C^{\kappa}, \delta_{\kappa}\right)_{\kappa}$ the universal complex of chiral singular fibers for proper $C^{\infty}$ stable maps of oriented 5-manifolds into 4-manifolds. Note that its unique cohomology group that makes sense is its third cohomology group, and is nothing but the kernel of the coboundary homomorphism $\delta_{3}$.

Then we get the following.

Proposition 8.1 The 3-dimensional cohomology group of the universal complex of chiral singular fibers for proper $C^{\infty}$ stable maps of oriented 5-manifolds into 4manifolds is an infinite cyclic group generated by the $C^{0}$ equivalence class modulo regular fibers of III $^{8}$ type fibers.

Proof Recall that we have exactly three $C^{0}$ equivalence classes modulo regular fibers of chiral singular fibers of codimension 3 , namely, $\mathrm{III}^{5}, \mathrm{III}^{7}$ and $\mathrm{III}^{8}$, by Proposition 6.1. By Lemma 6.3, the incidence coefficients involving $\mathrm{III}^{8}$ are all zero and hence III $^{8}$ is a cocycle. On the other hand, for the other two equivalence classes of chiral singular fibers, we have, for example,

$$
\begin{array}{ll}
{\left[\mathrm{III}^{5}: \mathrm{IV}^{11}\right] \neq 0,} & {\left[\mathrm{III}^{5}: \mathrm{IV}^{10}\right]=0,} \\
{\left[\mathrm{III}^{7}: \mathrm{IV}^{11}\right]=0,} & {\left[\mathrm{III}^{7}: \mathrm{IV}^{10}\right] \neq 0 .}
\end{array}
$$

Therefore, a linear combination of $\mathrm{III}^{5}, \mathrm{III}^{7}$ and $\mathrm{III}^{8}$ is a cocycle if and only if the coefficients of $\mathrm{III}^{5}$ and $\mathrm{III}^{7}$ both vanish. Therefore, the kernel of the coboundary homomorphism $\delta_{3}$ is infinite cyclic and is generated by III $^{8}$. This completes the proof.

According to Proposition 8.1, we can interpret our main theorem (Theorem 5.5) as follows. The 3-dimensional cohomology class represented by the cocycle III $^{8}$ of the universal complex of chiral singular fibers for proper $C^{\infty}$ stable maps of oriented 5-manifolds into 4-manifolds gives a complete invariant of the oriented cobordism 
class of the source 4-manifold. In particular, for $N=\mathbb{R}^{3}$, it gives a complete invariant of the oriented bordism class of a $C^{\infty}$ stable map of a closed oriented 4-manifold into $\mathbb{R}^{3}$.

For related discussions, see [24].

We also see that the fiber which satisfies the property as in Theorem 5.5 should necessarily be the fiber of type III $^{8}$. This explains the reason why the III $^{8}$ type fiber appeared in the modulo two Euler characteristic formula in [24] (see Corollary 5.6 of the present paper).

Remark 8.2 If we can realize the proof of our main theorem as mentioned in Remark 7.6 for arbitrary stable maps of closed oriented null-cobordant 4-manifolds into 3manifolds, then that would imply that $\mathrm{III}^{8}$ is a cocycle of the universal complex (see [24, Section 12.2]). That is, it might be possible to prove that $\mathrm{III}^{8}$ is a cocycle without even classifying the singular fibers.

\section{References}

[1] Y Ando, On local structures of the singularities $A_{k}, D_{k}$ and $E_{k}$ of smooth maps, Trans. Amer. Math. Soc. 331 (1992) 639-651 MR1055564

[2] T Bröcker, K Jänich, Introduction to differential topology, Cambridge University Press, Cambridge (1982) MR674117

[3] P E Conner, E E Floyd, Differentiable periodic maps, Ergebnisse series 33, SpringerVerlag, Berlin (1964) MR0176478

[4] J Damon, Topological properties of real simple germs, curves, and the nice dimensions $n>p$, Math. Proc. Cambridge Philos. Soc. 89 (1981) 457-472 MR602300

[5] C Ehresmann, Sur les espaces fibrés différentiables, C. R. Acad. Sci. Paris 224 (1947) 1611-1612 MR0020774

[6] H Endo, Meyer's signature cocycle and hyperelliptic fibrations, Math. Ann. 316 (2000) 237-257 MR1741270

[7] C G Gibson, K Wirthmüller, A A du Plessis, E J N Looijenga, Topological stability of smooth mappings, Lecture Notes in Mathematics 552, Springer-Verlag, Berlin (1976) MR0436203

[8] M Golubitsky, V Guillemin, Stable mappings and their singularities, Graduate Texts in Mathematics 14, Springer-Verlag, New York (1973) MR0341518

[9] M È Kazaryan, Hidden singularities and Vassiliev's homology complex of singularity classes, Mat. Sb. 186 (1995) 119-128 MR1376094 English translation in Sb. Math. 186 (1995) 1811-1820 
[10] L Kushner, H Levine, P Porto, Mapping three-manifolds into the plane I, Bol. Soc. Mat. Mexicana (2) 29 (1984) 11-33 MR790729

[11] K Lamotke, The topology of complex projective varieties after S Lefschetz, Topology 20 (1981) 15-51 MR592569

[12] H Levine, Classifying immersions into $\mathbf{R}^{4}$ over stable maps of 3-manifolds into $\mathbf{R}^{2}$, Lecture Notes in Mathematics 1157, Springer-Verlag, Berlin (1985) MR814689

[13] J N Mather, Stability of $C^{\infty}$ mappings V: Transversality, Advances in Math. 4 (1970) 301-336 (1970) MR0275461

[14] J N Mather, Stability of $C^{\infty}$ mappings VI: The nice dimensions, from: "Proceedings of Liverpool Singularities Symposium I (1969/70)", Lecture Notes in Mathematics 192, Springer-Verlag, Berlin (1971) 207-253 MR0293670

[15] Y Matsumoto, On 4-manifolds fibered by tori II, Proc. Japan Acad. Ser. A Math. Sci. 59 (1983) 100-103 MR711307

[16] Y Matsumoto, Lefschetz fibrations of genus two - a topological approach, from: "Topology and Teichmüller spaces (Katinkulta, 1995)", World Sci. Publishing, River Edge, NJ (1996) 123-148 MR1659687

[17] W Meyer, Die Signatur von Flächenbündeln, Math. Ann. 201 (1973) 239-264 MR0331382

[18] T Ohmoto, Vassiliev complex for contact classes of real smooth map-germs, Rep. Fac. Sci. Kagoshima Univ. Math. Phys. Chem. 27 (1994) 1-12 MR1341346

[19] T Ohmoto, O Saeki, K Sakuma, Self-intersection class for singularities and its application to fold maps, Trans. Amer. Math. Soc. 355 (2003) 3825-3838 MR1990176

[20] R Rimányi, A Szücs, Pontrjagin-Thom-type construction for maps with singularities, Topology 37 (1998) 1177-1191 MR1632908

[21] R Sadykov, Elimination of singularities of smooth mappings of 4-manifolds into 3manifolds, Topology Appl. 144 (2004) 173-199 MR2097135

[22] O Saeki, Topology of special generic maps of manifolds into Euclidean spaces, Topology Appl. 49 (1993) 265-293 MR1208678

[23] O Saeki, Fold maps on 4-manifolds, Comment. Math. Helv. 78 (2003) 627-647 MR1998397

[24] O Saeki, Topology of singular fibers of differentiable maps, Lecture Notes in Mathematics 1854, Springer-Verlag, Berlin (2004) MR2106689

[25] K Sakuma, On special generic maps of simply connected 2n-manifolds into $\mathbb{R}^{3}$, Topology Appl. 50 (1993) 249-261 MR1227553

[26] V A Vassiliev, Lagrange and Legendre characteristic classes, Advanced Studies in Contemporary Mathematics 3, Gordon and Breach Science Publishers, New York (1988) MR1065996 
[27] T Yamamoto, Classification of singular fibres of stable maps of 4-manifolds into 3-manifolds and its applications, J. Math. Soc. Japan (to appear)

[28] T Yamamoto, Classification of singular fibers and its applications, Master's thesis, Hokkaido University (2002) (in Japanese)

Faculty of Mathematics, Kyushu University

Hakozaki, Fukuoka 812-8581, Japan

Department of Mathematics, Hokkaido University

Sapporo 060-0810, Japan

saeki@math.kyushu-u.ac.jp, taku_chan@math.sci.hokudai.ac.jp

http://www.math.kyushu-u.ac.jp/ saeki/

Proposed: Yasha Eliashberg

Seconded: Shigeyuki Morita, Rob Kirby
Received: 8 October 2004

Revised: 22 February 2006 